\title{
Continuous unitary transformations in two-level boson systems
}

\author{
Sébastien Dusuel, ${ }^{1, *}$ Julien Vidal, ${ }^{2, \dagger}$ José M. Arias, ${ }^{3, \ddagger}$ Jorge Dukelsky,, ,§ and José Enrique García-Ramos ${ }^{5, \|}$ \\ ${ }^{1}$ Institut für Theoretische Physik, Universität zu Köln, Zülpicher Str. 77, D-50937 Köln, Germany \\ ${ }^{2}$ Laboratoire de Physique Théorique de la Matière Condensée, CNRS UMR 7600, Université Pierre et Marie Curie, 4 Place Jussieu, F-75252 \\ Paris Cedex 05, France \\ ${ }^{3}$ Departamento de Física Atómica, Molecular y Nuclear, Facultad de Física, Universidad de Sevilla, Apartado 1065, E-41080 Sevilla, Spain \\ ${ }^{4}$ Instituto de Estructura de la Materia, CSIC, Serrano 123, E-28006 Madrid, Spain \\ ${ }^{5}$ Departamento de Física Aplicada, Universidad de Huelva, E-21071 Huelva, Spain
}

(Received 28 September 2005; published 30 December 2005)

\begin{abstract}
Two-level boson systems displaying a quantum phase transition from a spherical (symmetric) to a deformed (broken) phase are studied. A formalism to diagonalize Hamiltonians with $O(2 L+1)$ symmetry for large number of bosons is worked out. Analytical results beyond the simple mean-field treatment are deduced by using the continuous unitary transformations technique. In this scheme, a $1 / N$ expansion for different observables is proposed and allows one to compute the finite-size scaling exponents at the critical point. Analytical and numerical results are compared and reveal the power of the present approach to compute the finite-size corrections in such a context.
\end{abstract}

DOI: 10.1103/PhysRevC.72.064332

PACS number(s): 21.60.Fw, 21.10.Re, 05.10.Cc, 75.40.Cx

\section{INTRODUCTION}

The study of two-level systems has been a topic of interest since the first steps in the development of quantum mechanics. The main advantage of these models is that they can be numerically diagonalized for very large dimensions and, at the same time, they can model realistic quantum many-body systems. Typical examples are the Jaynes-Cummings model of quantum optics [1], the vibron model (VM) of quantum chemistry [2], the two-level pairing model in condensed matter [3] and in nuclear physics [4], the Lipkin-Meshkov-Glick model (LMG) [5-7], and the interacting boson model (IBM) [8] of nuclear structure. Although some of these models describe two-level fermion systems, the model Hamiltonian can always be written in terms of $S U$ (2) pseudospin operators. Subsequently, the spin Hamiltonian can be expressed in terms of bosons using either the finite Schwinger representation or the infinite Holstein-Primakoff representation of the $S U(2)$ algebra. An example is the LMG model, which has recently been newly revived as a model of quantum spins with long-range interactions to investigate the relationship between entanglement and quantum phase transitions (QPTs) [9-15]. In its boson representation, it has also been recently used as a simplified model to describe the Josephson effect between two Bose-Einstein condensates [16].

In this work, we focus on finite two-level boson Hamiltonians, having the common feature that the lower level is always a scalar $L=0$ boson, hereafter written as $s$ boson. The upper level can have different multipolarities, generically noted as $L$, whose value defines a particular model. The LMG model

\footnotetext{
*Electronic address: sdusuel@thp.uni-koeln.de

†Electronic address: vidal@lptmc.jussieu.fr

${ }^{\ddagger}$ Electronic address: ariasc@us.es

§Electronic address: dukelsky@iem.cfmac.csic.es

"Electronic address: enrique.ramos@dfaie.uhu.es
}

in the Schwinger representation has a second scalar $(L=0)$ boson for the upper level. A dipolar $(L=1)$ boson leads to the VM and a quadrupolar $(L=2)$ boson corresponds to the IBM. Higher angular momentum bosons can lead new models, such as, for example, a model of octupole vibrations in terms of $s$ and octupolar $(L=3)$ bosons. A schematic representation of the model is shown in Fig. 1.

All these two-level boson models are governed by an algebraic structure that is constructed of all bilinear combinations of creation and annihilation boson operators that generate the algebra of $U(2 L+2)$. One of the main features of these models is that one can construct dynamical symmetries in which the Hamiltonian can be written in terms of Casimir invariants of a nested chain of subalgebras of $U(2 L+2)$. In these particular cases, the problem is analytically solvable providing a powerful tool to check approximate methods and a reference for more detailed calculations. In addition, if the Hamiltonian is written as a linear combination of Casimir invariants of the subalgebras $U(2 L+1)$ and $O(2 L+2)$ the model is still quantum integrable but then requires solving Bethe-like equations numerically [17]. The exact solution, given by Richardson almost 40 years ago [18], is reduced to a set of $M$ nonlinear coupled equations, where $M$ is the number of boson pairs. For two-level boson models it turns out that the numerical diagonalization of the Hamiltonian presented below is more efficient than solving the Richardson equations.

The aim of this work is to study the QPT that occurs in the two-level boson system as it evolves from the spherical vibrational $U(2 L+1)$ symmetry to the deformed $O(2 L+2)$ symmetry, as a function of a control parameter. Although, strictly speaking, QPTs are defined for macroscopic systems, there is a renewed interest in studying structural changes in finite-size systems as the precursors of a QPT in the thermodynamic limit. Traces of these QPTs are readily observed in finite systems and their properties are then correlated with the idealized thermodynamic system [19]. The understanding of 


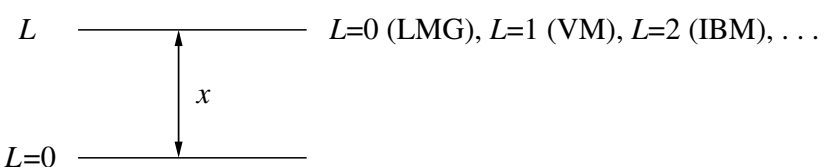

FIG. 1. Schematic representation of the two-level boson model studied in this article.

the modifications on the characteristics of the QPT induced by finite-size effects is of crucial importance to extend the concept of phase transitions to finite systems. Several techniques have long been used to extrapolate numerical results obtained by large-scale diagonalizations or Monte Carlo calculations to the infinite system. Here, we focus on a somewhat inverse problem, which is the finite-size corrections to the observables in two-level boson models such as the ground-state energy, the gap, the occupation number, and some electromagnetic transition rates. Although the zeroth order in the boson number $N$ is given by the Hartree mean-field approach for the ground state and the random phase approximation for the excited states, going beyond this order implies the use of more sophisticated techniques. We make use here of the continuous unitary transformations (CUTs) and give the first $1 / N$ corrections to the observables in the whole $U(2 L+1)$ to $O(2 L+2)$ transition.

The structure of the article is the following. In Sec. II we introduce the two-level boson models and the formalism for the numerical diagonalization for a very large number of bosons. Section III describes the mean-field treatment of the two-level boson models. Section IV is devoted to the study of the symmetric phase using CUTs. Analytical expressions for different orders in the $1 / N$ expansion of the ground-state energy, the gap, the expectation value of the number of $L$ bosons in the ground state, and the transition matrix element between the ground state and the first excited state are obtained. In Sec. V the broken phase is analyzed, and in Sec. VI the study of the critical point is presented from the spherical phase. In this section, we obtain the finite-size scaling exponents for the quantities cited above by analyzing the divergence of their $1 / N$ expansion. In Sec. VII a comparison of the numerical results obtained using the formalism presented in Sec. II with the analytical CUTs results is presented. Section VIII is for summary and conclusions. Technical details concerning flow equations can be found in appendices.

\section{TWO-LEVEL BOSON MODELS}

In this section, we present a simple algorithm for diagonalizing boson Hamiltonians with $O(2 L+1)$ symmetry for large boson numbers. The formalism is based on previous studies $[20,21]$ and is a generalization of the one presented recently for treating the IBM [22].

We consider the following boson pairing Hamiltonian

$$
H=x n_{L}+\frac{1-x}{4(N-1)}\left(P_{L}^{\dagger} P_{L}+P_{s}^{\dagger} P_{s}-P_{L}^{\dagger} P_{s}-P_{s}^{\dagger} P_{L}\right),
$$

with

$$
\begin{aligned}
& n_{L}=\sum_{\mu=-L}^{+L} L_{\mu}^{\dagger} L_{\mu}, \\
& P_{s}^{\dagger}=\left(P_{s}\right)^{\dagger}=s^{\dagger} s^{\dagger}, \\
& P_{L}^{\dagger}=\left(P_{L}\right)^{\dagger}=\sum_{\mu=-L}^{+L}(-1)^{\mu} L_{\mu}^{\dagger} L_{-\mu}^{\dagger} .
\end{aligned}
$$

$L_{\mu}^{\dagger}$ creates a boson in the excited $L$ level with projection $\mu$, whereas $L_{\mu}$ destroys it. We have introduced above the pair $P_{\rho}^{\dagger}$ operators with $\rho=s$ or $L$, which is used later on. The total number of bosons $N=n_{s}+n_{L}$ is a conserved quantity. For $x=0, H$ can be cast into a linear combination of the quadratic Casimir operators of $O(2 L+2)$ and the corresponding subalgebras, whereas for $x=1, H$ is the linear Casimir operator of the $U(2 L+1)$ algebra. Here, $x$ plays the role of a control parameter, driving the system from the $O(2 L+2)$ deformed phase to the $U(2 L+1)$ spherical phase.

The boson pairing Hamiltonian (1) can be studied in an elegant way by means of the noncompact $S U(1,1)$ algebra of boson pair operators. For the subspace of $\rho$ bosons, where $\rho$ stands generically either for $s$ or $L$ bosons, the $S U(1,1)$ generators are the raising operator $K_{\rho}^{+}$, the lowering operator $K_{\rho}^{-}=\left(K_{\rho}^{+}\right)^{\dagger}$ and the Cartan operator $K_{\rho}^{0}$ defined as

$$
\begin{aligned}
K_{\rho}^{+} & =\frac{1}{2} P_{\rho}^{\dagger}, \\
K_{\rho}^{-} & =\frac{1}{2} P_{\rho}, \\
K_{\rho}^{0} & =\frac{1}{2} \sum_{\mu}\left(\rho_{\mu}^{\dagger} \rho_{\mu}+\frac{1}{2}\right)=\frac{1}{2} n_{\rho}+\frac{1}{4} D_{\rho},
\end{aligned}
$$

with $D_{\rho}=2 \rho+1$. The three operators $\left\{K_{\rho}^{+}, K_{\rho}^{-}, K_{\rho}^{0}\right\}$ satisfy the $S U(1,1)$ commutator algebra

$$
\begin{aligned}
{\left[K_{\rho}^{0}, K_{\rho^{\prime}}^{ \pm}\right] } & = \pm \delta_{\rho, \rho^{\prime}} K_{\rho}^{ \pm}, \\
{\left[K_{\rho}^{+}, K_{\rho^{\prime}}^{-}\right] } & =-2 \delta_{\rho, \rho^{\prime}} K_{\rho}^{0} .
\end{aligned}
$$

The Casimir operator is

$$
C_{\rho}^{2}=\frac{1}{2}\left(K_{\rho}^{+} K_{\rho}^{-}+K_{\rho}^{-} K_{\rho}^{+}\right)-\left(K_{\rho}^{0}\right)^{2}=-\frac{D_{\rho}}{4}\left(\frac{D_{\rho}}{4}-1\right) .
$$

The complete set of eigenstates of the pairing Hamiltonian (1) can be constructed as a direct product of subspaces associated to $s$ and $L$ bosons. Each subspace can be written in terms of the rising operators $K_{\rho}^{+}$acting on the corresponding subspace of unpaired $\rho$ bosons

$$
\left|\tilde{n}_{\rho}, v_{\rho}\right\rangle=\frac{1}{\sqrt{C_{\rho, v_{\rho}}^{\tilde{n}_{\rho}}}} K_{\rho}^{+\tilde{n}_{\rho}}\left|\tilde{n}_{\rho}=0, v_{\rho}\right\rangle,
$$

where $v_{s}=v_{L=0}=0,1$ and $v_{L \neq 0}=0,1,2, \ldots$ The quantity $v_{\rho}$ is known as the boson seniority for $\rho$ bosons and gives the number of bosons of type $\rho$ not coupled in pairs to zero. Note that from now on the label $\tilde{n}$ means number of boson 
pairs coupled to zero angular momentum. The total number of bosons is $2 \tilde{n}_{s}+2 \tilde{n}_{L}+v_{s}+v_{L}$. The normalization constant in Eq. (11) can be obtained from the action of $K_{\rho}^{-}$and $K_{\rho}^{0}$ on the $\rho$ subspace $\left|\tilde{n}_{\rho}=0, v_{\rho}\right\rangle$

$$
\begin{aligned}
& K_{\rho}^{-}\left|\tilde{n}_{\rho}=0, v_{\rho}\right\rangle=0, \\
& K_{\rho}^{0}\left|\tilde{n}_{\rho}=0, v_{\rho}\right\rangle=\left(\frac{1}{2} v_{\rho}+\frac{1}{4} D_{\rho}\right)\left|\tilde{n}_{\rho}=0, v_{\rho}\right\rangle,
\end{aligned}
$$

and the commutation relation

$$
\left[\left[K_{\rho}^{-}, K_{\rho}^{+}\right], K_{\rho}^{+}\right]=2 K_{\rho}^{+},
$$

then

$$
\begin{aligned}
& K_{\rho}^{-}\left(K_{\rho}^{+}\right)^{\tilde{n}_{\rho}}\left|\tilde{n}_{\rho}=0, v_{\rho}\right\rangle \\
& \quad=\tilde{n}_{\rho}\left(\tilde{n}_{\rho}+\frac{D_{\rho}}{2}+v_{\rho}-1\right)\left(K_{\rho}^{+}\right)^{\tilde{n}_{\rho}-1}\left|\tilde{n}_{\rho}=0, v_{\rho}\right\rangle,
\end{aligned}
$$

and

$$
\begin{aligned}
\left\langle\tilde{n}_{\rho}, v_{\rho} \mid \tilde{n}_{\rho}, v_{\rho}\right\rangle= & \frac{\tilde{n}_{\rho}}{2}\left(2 \tilde{n}_{\rho}+2 \rho+2 v_{\rho}-1\right) \\
& \times\left\langle\tilde{n}_{\rho}-1, v_{\rho} \mid \tilde{n}_{\rho}-1, v_{\rho}\right\rangle,
\end{aligned}
$$

and finally

$$
\begin{aligned}
\left\langle\tilde{n}_{\rho}, v_{\rho} \mid \tilde{n}_{\rho}, v_{\rho}\right\rangle & =\frac{\tilde{n}_{\rho} !\left(2 \tilde{n}_{\rho}+2 \rho+2 v_{\rho}-1\right) ! !}{2^{\tilde{n}_{\rho}}\left(2 \rho+2 v_{\rho}-1\right) ! !} \\
& =C_{\rho, v_{\rho}}^{\tilde{n}_{\rho}} .
\end{aligned}
$$

Remember that the label $\rho$ stands for $s$ or $L$ bosons and takes the following numerical values: 0 for $s$ bosons and $L$ for $L$ bosons. Once the basis for each subspace is obtained [Eqs. (11) and (17)], the complete basis set for the pairing Hamiltonian [Eq. (1)] is easily constructed,

$$
\begin{aligned}
\left|\tilde{n}_{s}, v_{s} ; \tilde{n}_{L}, v_{L}\right\rangle= & \frac{1}{\sqrt{C_{s, v_{s}}^{\tilde{n}_{s}} C_{L, v_{L}}^{\tilde{n}_{L}}}}\left(K_{s}^{+}\right)^{\tilde{n}_{s}}\left(K_{L}^{+}\right)^{\tilde{n}_{L}} \\
& \times\left|\tilde{n}_{s}=0, v_{s} ; \tilde{n}_{L}=0, v_{L}\right\rangle .
\end{aligned}
$$

We now diagonalize the Hamiltonian given in Eq. (1) in the basis of the states given by Eq. (18). Note that in the following $n$ refers to boson number operators but $\tilde{n}$ are number of boson pairs. The relevant matrix elements for the construction of the Hamiltonian matrix are as follows:

$$
\begin{gathered}
\left\langle\tilde{n}_{s}, v_{s} ; \tilde{n}_{L}, v_{L}\left|n_{s}\right| \tilde{n}_{s}, v_{s} ; \tilde{n}_{L}, v_{L}\right\rangle=2 \tilde{n}_{s}+v_{s}, \\
\left\langle\tilde{n}_{s}, v_{s} ; \tilde{n}_{L}, v_{L}\left|n_{L}\right| \tilde{n}_{s}, v_{s} ; \tilde{n}_{L}, v_{L}\right\rangle=2 \tilde{n}_{L}+v_{L}, \\
\left\langle\tilde{n}_{s}, v_{s} ; \tilde{n}_{L}, v_{L}\left|K_{s}^{+} K_{s}^{-}\right| \tilde{n}_{s}, v_{s} ; \tilde{n}_{L}, v_{L}\right\rangle=\tilde{n}_{s}\left(\tilde{n}_{s}+v_{s}-\frac{1}{2}\right), \\
\left\langle\tilde{n}_{s}, v_{s} ; \tilde{n}_{L}, v_{L}\left|K_{L}^{+} K_{L}^{-}\right| \tilde{n}_{s}, v_{s} ; \tilde{n}_{L}, v_{L}\right\rangle=\tilde{n}_{L}\left(\tilde{n}_{L}+L+v_{L}-\frac{1}{2}\right), \\
\left\langle\left(\tilde{n}_{s}-1\right), v_{s} ;\left(\tilde{n}_{L}+1\right), v_{L}\left|K_{L}^{+} K_{s}^{-}\right| \tilde{n}_{s}, v_{s} ; \tilde{n}_{L}, v_{L}\right\rangle \\
=\frac{1}{2} \sqrt{\tilde{n}_{s}\left(\tilde{n}_{L}+1\right)\left(2 \tilde{n}_{s}+2 v_{s}-1\right)\left(2 \tilde{n}_{L}+2 L+2 v_{L}+1\right) .}
\end{gathered}
$$

It is clear that the Hamiltonian does not mix states with different boson seniority quantum numbers. Thus, the
Hamiltonian matrix is block diagonal. In addition, within one block the matrix is tridiagonal, making the diagonalization simple. The different states are obtained as follows: one starts with the boson subspace containing $N / 2$ boson pairs coupled to zero angular momentum, $v_{s}=v_{L}=0$. The diagonalization of $H$ in this subspace provides states with angular momentum zero and the first eigenstate is the ground state. Next, one goes to the block with one broken boson pair. This block is composed of two separate blocks because the two bosons can be one $s$ boson and one $L$ boson $\left(v_{s}=1, v_{L}=1\right)$ or two $L$ bosons not coupled to zero because the coupling to zero is included in the first block, $\left(v_{s}=0, v_{L}=2\right)$. Notice that two unpaired $s$ bosons are not possible because they are always coupled to zero and consequently they are counted in the first block. For the case of the LMG model in which $L=0$, the block $v_{s}=0, v_{L}=2$ is not allowed for the same reason. The block $v_{s}=1, v_{L}=1$ provides states with angular momentum $L$, the first of them is the first excited state of the system. The block $v_{s}=0, v_{L}=2$ contains states with two $L$ bosons not coupled to zero angular momentum; it contains angular momenta: $2 L, 2 L-2, \ldots, 2$. Next, there is another block with two broken boson pairs composed of two subblocks: $v_{s}=1, v_{L}=3$ and $v_{s}=0, v_{L}=4$. Again, the block $v_{s}=0$, $v_{L}=4$ is absent for the LMG model. This construction continues for $3,4, \ldots, N / 2$ broken boson pairs. Few first low-lying Hamiltonian eigenstates are depicted schematically in Fig. 2.

Direct block diagonalization of the Hamiltonian in the basis of the states given by Eq. (18) provides observables as the ground-state energy or the gap and also the wave functions of the states. With the wave function of the ground state the expectation value of the number of $L$ bosons in the ground state can be easily calculated. One can also calculate the transition probability from the ground state to the first excited state provided with the appropriate operator. The natural transition operator for the pairing Hamiltonian we are considering is as follows:

$$
T_{L_{\mu}}=L_{\mu}^{\dagger} s+(-1)^{\mu} s^{\dagger} L_{-\mu}
$$

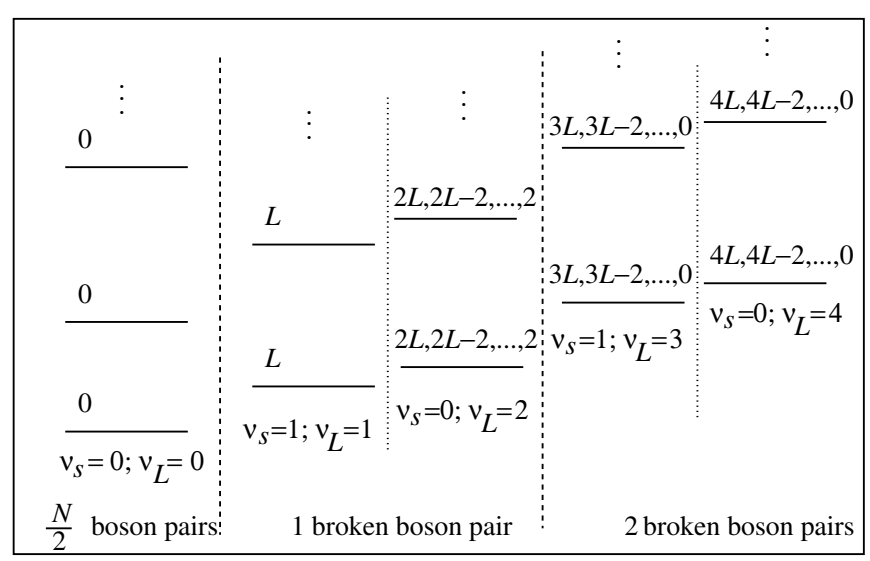

FIG. 2. Schematic representation of the level sequence obtained by diagonalizing the Hamiltonian for the two-level boson models studied in this article. Numbers above the lines are angular momenta. 
The action of $T_{L_{\mu}}$ on the subspace $v_{s}=0, v_{L}=0$ that includes the ground state is as follows:

$$
\begin{aligned}
& T_{L_{\mu}}\left|\tilde{n}_{s}, 0 ; \tilde{n}_{L}, 0\right\rangle \\
& =\frac{1}{\sqrt{C_{s, 0}^{\tilde{n}_{s}} C_{L, 0}^{\tilde{n}_{L}}}}\left[\tilde{n}_{s}\left(K_{s}^{+}\right)^{\tilde{n}_{s}-1}\left(K_{L}^{+}\right)^{\tilde{n}_{L}} s^{\dagger} L_{\mu}^{\dagger}+\tilde{n}_{L}\right. \\
& \left.\quad \times\left(K_{s}^{+}\right)^{\tilde{n}_{s}}\left(K_{L}^{+}\right)^{\tilde{n}_{L}-1} s^{\dagger} L_{\mu}^{\dagger}\right]\left|\tilde{n}_{s}=0,0 ; \tilde{n}_{L}=0,0\right\rangle,
\end{aligned}
$$

where the state $\left.\left|\tilde{n}_{s}=0, v_{s}=0 ; \tilde{n}_{L}=0, v_{L}=0\right\rangle \equiv \mid 0\right)$ is the boson vacuum.

Then, the matrix elements of $T_{L_{\mu}}$ connecting the subspaces $v_{s}=0, v_{L}=0$ and $v_{s}=1, v_{L}=1$ (which includes the first excited state) are as follows:

$$
\begin{aligned}
& \left\langle\left(\tilde{n}_{s}-1\right), v_{s}=1 ; \tilde{n}_{L}, v_{L}=1\left|T_{L_{\mu}}\right| \tilde{n}_{s}, v_{s}=0 ; \tilde{n}_{L}, v_{L}=0\right\rangle \\
& \quad=\sqrt{\frac{2 \tilde{n}_{s}\left(2 \tilde{n}_{L}+2 L+1\right)}{2 L+1}} \\
& \left\langle\tilde{n}_{s}, v_{s}=1 ;\left(\tilde{n}_{L}-1\right), v_{L}=1\left|T_{L_{\mu}}\right| \tilde{n}_{s}, v_{s}=0 ; \tilde{n}_{L}, v_{L}=0\right\rangle \\
& \quad=\sqrt{\frac{2 \tilde{n}_{L}\left(2 \tilde{n}_{s}+1\right)}{2 L+1}} .
\end{aligned}
$$

If we write each eigenstate of the Hamiltonian as

$$
\left|\Psi_{i}, v_{s}, v_{L}\right\rangle=\sum_{\tilde{n}_{s}, \tilde{n}_{L}} c_{\tilde{n}_{s}, \tilde{n}_{L}}^{v_{s}, v_{L}}\left|\tilde{n}_{s}, v_{s} ; \tilde{n}_{L}, v_{L}\right\rangle,
$$

the matrix element of the $T_{L \mu}$ operator between the ground state $|0\rangle \equiv\left|\boldsymbol{\Psi}_{0}, 0,0\right\rangle$ and the first excited state $|1\rangle \equiv\left|\Phi_{0}, 1,1\right\rangle$ is

$$
\begin{aligned}
\left\langle 1\left|T_{L \mu}\right| 0\right\rangle= & \sum_{\tilde{n}_{s}, \tilde{n}_{L}}\left[\sqrt{\frac{2 \tilde{n}_{s}\left(2 \tilde{n}_{L}+2 L+1\right)}{2 L+1}} c_{\tilde{n}_{s}, 0, \tilde{n}_{L}}^{0,0} c_{\tilde{n}_{s}-1, \tilde{n}_{L}}^{1,1}\right. \\
& \left.+\sqrt{\frac{2 \tilde{n}_{L}\left(2 \tilde{n}_{s}+1\right)}{2 L+1}} c_{\tilde{n}_{s}, \tilde{n}_{L}}^{0,0} c_{\tilde{n}_{s}, \tilde{n}_{L}-1}^{1,1}\right] .
\end{aligned}
$$

The formalism presented in this section provides the exact full solution of the problem. A simpler approach to study ground-state properties in the large $N$ limit is provided by the mean-field analysis presented in the next section. This limit is a good benchmark to test more elaborate results.

\section{MEAN-FIELD ANALYSIS}

The geometrical interpretation of the Hamiltonian [Eq. (1)] can be obtained by introducing a Hartree axial coherent state that allows us to associate to it a geometrical shape in terms of a deformation variable $\beta$. For a system with $N$ bosons, this state is obtained by acting $N$ times with a condensed boson $\Gamma^{\dagger}$ on the boson vacuum $\mid 0$ )

$$
\left.|N, \beta\rangle=\frac{1}{\sqrt{N !}}\left(\Gamma^{\dagger}\right)^{N} \mid 0\right),
$$

where the basic condensed boson operator is given by the following:

$$
\Gamma^{\dagger}=\frac{1}{\sqrt{1+\beta^{2}}}\left(s^{\dagger}+\beta L_{0}^{\dagger}\right)
$$

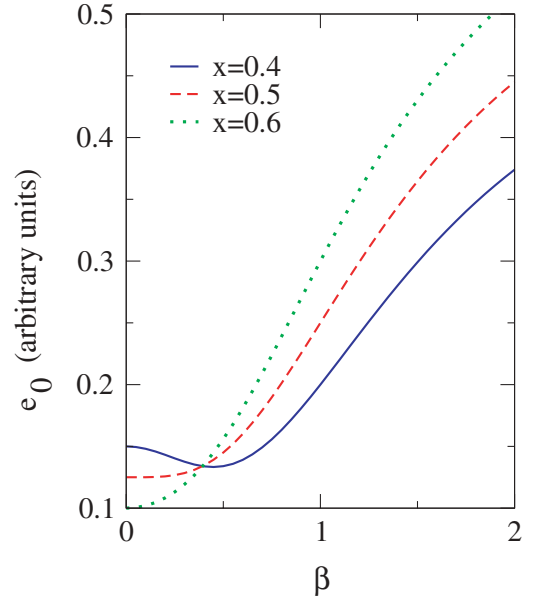

FIG. 3. (Color online) Energy surfaces per boson for the Hamiltonian [Eq. (1)] for different values of the control parameter $x$.

which depends on the $\beta$ shape variable. The energy surface is defined as the expectation value of $H$ in the intrinsic state

$$
\begin{aligned}
E(N, \beta) & =\langle N, \beta|H| N, \beta\rangle \\
& =N\left[x \frac{\beta^{2}}{1+\beta^{2}}+\frac{1-x}{4}\left(\frac{1-\beta^{2}}{1+\beta^{2}}\right)^{2}\right] .
\end{aligned}
$$

Minimizing the variational energy $E(N, \beta)$ with respect to $\beta$ leads to a critical point at $x_{c}=0.5$. For $x>x_{c}$ (symmetric phase), the ground state is spherical and is obtained for $\beta=0$, whereas for $x<x_{c}$ (broken phase) it is deformed since the minimum of the energy per boson $e_{0}=E(N, \beta) / N$ is obtained for $\beta=\sqrt{1-2 x}$ as can be seen in Fig. 3. At the critical point, it is worth noting that the energy surface is a flat $\beta^{4}$ surface near $\beta=0$ [22,23]. Within this mean-field (variational) approach, one thus gets the following ground-state energy per boson:

$$
\begin{aligned}
& e_{0}\left(x \geqslant x_{c}\right)=\frac{1-x}{4}, \\
& e_{0}\left(x \leqslant x_{c}\right)=\frac{x}{4} \frac{2-3 x}{1-x} .
\end{aligned}
$$

One can also straightforwardly compute the expectation value of $n_{L}$ in the ground state, which is found to vanish in the symmetric phase and equals

$$
\left\langle n_{L}\right\rangle=N \frac{1-2 x}{2(1-x)},
$$

in the broken one.

However, other properties, as excitation energies or transition probabilities, that imply excited states require to go one step beyond this simple mean-field level. In the following, we use a combination of several methods already detailed for the simple case $L=0$ in Ref. [24] that allow us to compute the corrections to these mean-field results as well as the gap or the transition rates that require the knowledge of excited states. 


\section{THE SYMMETRIC PHASE $(1 / 2<x<1)$}

The starting point of our analysis is the elimination of the $s$ boson by means of a Holstein-Primakoff boson expansion [25] of $s$ and $L$ bosons (for a review in boson expansion techniques see Ref. [26]). Therefore, we introduce a set of $b_{\mu}$ bosons such that the mapping

$$
\begin{aligned}
L_{\mu}^{\dagger} L_{v} & =b_{\mu}^{\dagger} b_{v}, \\
L_{\mu}^{\dagger} s & =N^{1 / 2} b_{\mu}^{\dagger}\left(1-n_{b} / N\right)^{1 / 2}=\left(s^{\dagger} L_{\mu}\right)^{\dagger}, \\
s^{\dagger} s & =N-n_{b} .
\end{aligned}
$$

fulfills the commutation relations at each order in $N$ in the Taylor expansion of the square root.

With these notations, we have the following:

$$
\begin{aligned}
n_{L} & =n_{b}, \\
P_{L}^{\dagger} P_{L} & =P_{b}^{\dagger} P_{b}, \\
P_{s}^{\dagger} P_{s} & =(N-1)\left(N-2 n_{b}\right)+: n_{b}^{2}:, \\
P_{L}^{\dagger} P_{s} & =\sum_{\mu}(-1)^{\mu} L_{\mu}^{\dagger} s L_{-\mu}^{\dagger} s, \\
& =N \sum_{\mu}(-1)^{\mu} b_{\mu}^{\dagger}\left(1-n_{b} / N\right)^{1 / 2} b_{-\mu}^{\dagger}\left(1-n_{b} / N\right)^{1 / 2},
\end{aligned}
$$

where: $A$ : denotes the normal-ordered form of the operator $A$.

The Holstein-Primakoff mapping eliminates the $s$ boson at the cost of introducing infinitely many boson terms. However, each term in the expansion has a definite $1 / N$ order. As shown in the preceding section, for $1 / 2<x<1$, the number of $L$ boson in the ground state goes to zero in the thermodynamical limit. Thus, to capture the finite $N$ corrections, one performs a $1 / N$ expansion of the Hamiltonian considering that $n_{b} / N \ll 1$. In this phase, the Hamiltonian [Eq. (1)] reads as follows:

$$
\begin{aligned}
H= & N^{1}\left(\frac{1-x}{4}\right)+N^{0}\left(\frac{1-x}{4}\right) \\
& \times\left[\frac{2(3 x-1)}{1-x} n_{b}-\left(P_{b}^{\dagger}+P_{b}\right)\right]+N^{-1}\left(\frac{1-x}{4}\right) \\
& \times\left[: n_{b}^{2}:+P_{b}^{\dagger} P_{b}-\frac{1}{2}\left(P_{b}^{\dagger}+P_{b}\right)+P_{b}^{\dagger} n_{b}+n_{b} P_{b}\right] \\
& +N^{-2}\left(\frac{1-x}{4}\right) \\
& \times\left[: n_{b}^{2}:+P_{b}^{\dagger} P_{b}-\frac{3}{8}\left(P_{b}^{\dagger}+P_{b}\right)+P_{b}^{\dagger} n_{b}+n_{b} P_{b}\right] \\
& +O\left(1 / N^{3}\right) .
\end{aligned}
$$

Here, we have restricted this expansion to order $(1 / N)^{2}$ but the method we used can, in principle, be applied beyond this limit as shown in Ref. [24] for the LMG model $(L=0)$. Our aim is to diagonalize $H$ order by order. At the leading order, one obviously recovers the mean-field ground-state energy per boson $e_{0}(N)=(1-x) / 4+O(1 / N)$. At order $(1 / N)^{0}$, the Hamiltonian is quadratic and it can thus be easily diagonalized through a Bogoliubov transform, giving rise to the boson random phase approximation formalism presented in Ref. [27] and more recently exploited to describe the properties of the symmetric and broken-symmetry phases in the interacting boson model $[21,28]$. Higher-order terms cannot be diagonalized by a Bogoliubov transformation, so that one has to resort a more sophisticated method.

\section{A. CUTs formalism}

The CUTs technique has been conjointly proposed by Wegner [29] and Głazek and Wilson [30,31]. For a pedagogical introduction, we refer the reader to Refs. [32,33]. Here we only sketch the main lines of this simple and powerful approach.

The idea of the CUTs is to diagonalize the Hamiltonian in a continuous way starting from the original (bare) Hamiltonian $H=H(l=0)$. A flowing Hamiltonian is thus defined by the following:

$$
H(l)=U^{\dagger}(l) H(0) U(l),
$$

where $l$ is a scaling parameter such that $H(l=\infty)$ is diagonal, and $U(l)$ is a unitary transformation, i.e., satisfying $U(l) U^{\dagger}(l)=U^{\dagger}(l) U(l)=1$. Taking the derivative of Eq. (44) with respect to $l$ yields the differential (flow) equation

$$
\partial_{l} H(l)=[\eta(l), H(l)],
$$

where the generator of the unitary transformation $\eta(l)$ is

$$
\eta(l)=\partial_{l} U^{\dagger}(l) U(l)=-U^{\dagger}(l) \partial_{l} U(l) .
$$

CUTs are also a powerful tool to compute the expectation value of any observable $\Omega$. As for the Hamiltonian, we define a flowing operator

$$
\Omega(l)=U^{\dagger}(l) \Omega(0) U(l),
$$

which obeys

$$
\partial_{l} \Omega(l)=[\eta(l), \Omega(l)],
$$

with $\Omega=\Omega(l=0)$. The expectation value of $\Omega$ on an eigenstate $|\psi\rangle$ of $H$ is then given by the following:

$$
\langle\psi|\Omega| \psi\rangle=\left\langle\psi\left|U(l=\infty) \Omega(l=\infty) U^{\dagger}(l=\infty)\right| \psi\right\rangle,
$$

where $U^{\dagger}(l=\infty)|\psi\rangle$ is simply the eigenstate of the diagonal Hamiltonian $H(l=\infty)$.

The key point of this approach is an appropriate choice of the generator $\eta$, which, in fact, depends on the problem under consideration. Here, the Hamiltonian $H$ expressed in terms of $b$ boson can be schematically written as follows:

$$
H(0)=H_{0}(0)+H_{1}^{+}(0)+H_{1}^{-}(0)+H_{2}^{+}(0)+H_{2}^{-}(0),
$$

where $H_{1,2}^{-}=\left(H_{1,2}^{+}\right)^{\dagger}$ and 0,1 , or 2 subscripts indicate the number of created $(+)$ or annihilated $(-)$ excitations.

To perform the CUTs, we choose the so-called quasiparticle conserving generator first proposed by Mielke [34] in the context of finite matrices and generalized to many-body problems by Knetter and Uhrig [35], which reads

$$
\eta(l)=H_{1}^{+}(l)-H_{1}^{-}(l)+H_{2}^{+}(l)-H_{2}^{-}(l) .
$$

In the symmetric phase $\left(H_{1}^{ \pm}=0\right)$ this choice coincides with the generator proposed by Stein [36]. The flow equations are 
then simple quadratic functions of the Hamiltonians:

$$
\begin{aligned}
\partial_{l} H_{0}(l) & =2\left(\left[H_{1}^{+}(l), H_{1}^{-}(l)\right]+\left[H_{2}^{+}(l), H_{2}^{-}(l)\right]\right), \\
\partial_{l} H_{1}^{+}(l) & =\left[H_{1}^{+}(l), H_{0}(l)\right]+2\left[H_{2}^{+}(l), H_{1}^{-}(l)\right], \\
\partial_{l} H_{2}^{+}(l) & =\left[H_{2}^{+}(l), H_{0}(l)\right] .
\end{aligned}
$$

In the limit $l=\infty$, the Hamiltonian conserves the number of $b$ boson so that $H_{1,2}^{ \pm}(\infty)=0$ and $H(\infty)=H_{0}(\infty)$. Following the method developed for the LMG model in Ref. [14,24], we convert these equations, which deal with operators, into equations involving coupling constants. This is achieved by expanding Hamiltonians $H_{0}$ and $H_{1,2}^{ \pm}$in powers of $1 / N$ (see Sec. IV B).

\section{B. Flow equations for the Hamiltonian}

In the symmetric phase $\left(H_{1}^{ \pm}=0\right)$, we have three elementary operators : $n_{b}:, P_{b}^{\dagger}, P_{b}$ from which $H_{0}$ and $H_{2}^{ \pm}$are built. More precisely, the $1 / N$ expansion of these Hamiltonians can be written as follows:

$$
\begin{gathered}
H_{0}(l)=\sum_{\alpha, \beta, \delta \in \mathbb{N}} \frac{h_{0, \alpha, \beta}^{(\delta)}(l) P_{b}^{\dagger^{\beta}}: n_{b}^{\alpha}: P_{b}^{\beta}}{N^{\alpha+2 \beta+\delta-1}}, \\
H_{2}^{+}(l)=\sum_{\alpha, \beta, \delta \in \mathbb{N}} \frac{h_{2, \alpha, \beta}^{(\delta)}(l) P_{b}^{\dagger} P_{b}^{\dagger \beta}: n_{b}^{\alpha}: P_{b}^{\beta}}{N^{\alpha+2 \beta+\delta}} .
\end{gathered}
$$

Note that for $L=0$, one has $P_{b}^{\dagger} P_{b}=: n_{b}^{2}:$, so that $h_{k, \alpha, \beta}^{(\delta)}=$ $h_{k, \alpha+2 \beta, 0}^{(\delta)}$. One then readily recovers expressions given in Ref. [24] for the case of a scalar $b$ boson. Using this expansion and Eqs. (52)-(54), we can easily derive the flow equations for the couplings $h_{k, \alpha, \beta}^{(\delta)}(l)$, which are given in Appendix A up to order $(1 / N)^{2}$. These flow equations can be solved exactly and, at order $(1 / N)^{2}$, one finally obtains the following:

$$
\begin{aligned}
H(\infty)= & h_{0,0,0}(\infty)+h_{0,1,0}(\infty) n_{b}+h_{0,2,0}(\infty): n_{b}^{2}: \\
& +h_{0,0,1}(\infty) P_{b}^{\dagger} P_{b}+h_{0,3,0}(\infty): n_{b}^{3}: \\
& +h_{0,1,1}(\infty) P_{b}^{\dagger} n_{b} P_{b}+O\left(1 / N^{3}\right)
\end{aligned}
$$

with

$$
h_{0, \alpha, \beta}(l)=\sum_{\delta \in \mathbb{N}} \frac{h_{0, \alpha, \beta}^{(\delta)}(l)}{N^{\alpha+2 \beta+\delta-1}}
$$

and

$$
\begin{aligned}
h_{0,0,0}^{(0)}(\infty)= & \frac{1-x}{4}, \\
h_{0,0,0}^{(1)}(\infty)= & \frac{2 L+1}{2}\left[\frac{1-3 x}{2}+\Xi(x)^{1 / 2}\right], \\
h_{0,0,0}^{(2)}(\infty)= & (2 L+1)(1-x) x \\
& \times\left[\frac{-(2 L+5)+(6 L+13) x}{16 \Xi(x)}-\frac{L+2}{4 \Xi(x)^{1 / 2}}\right],
\end{aligned}
$$

$$
\begin{aligned}
h_{0,0,0}^{(3)}(\infty)= & -(2 L+1)(1-x) x^{2}\left[\frac{(2 L+1)-\left(8 L^{2}+6 L-33\right) x+\left(32 L^{2}-2 L-149\right) x^{2}-\left(24 L^{2}-38 L-179\right) x^{3}}{128 \Xi(x)^{5 / 2}}\right. \\
& \left.-\frac{2 L+5+\left(2 L^{2}-3 L-17\right) x-\left(2 L^{2}-5 L-20\right) x^{2}}{16 \Xi(x)^{2}}\right],
\end{aligned}
$$$$
h_{0,1,0}^{(1)}(\infty)=(1-x) x\left[\frac{-1+(2 L+5) x}{4 \Xi(x)}-\frac{L+2}{2 \Xi(x)^{1 / 2}}\right] \text {, }
$$

$$
h_{0,1,0}^{(0)}(\infty)=\Xi(x)^{1 / 2},
$$

$$
\begin{aligned}
h_{0,1,0}^{(2)}(\infty)= & -(1-x) x^{2}\left[\frac{L+1-\left(2 L^{2}+3 L-6\right) x+\left(12 L^{2}+15 L-23\right) x^{2}-\left(10 L^{2}+5 L-32\right) x^{3}}{16 \Xi(x)^{5 / 2}}\right. \\
& \left.-\frac{1+\left(2 L^{2}+5 L-1\right) x-\left(2 L^{2}+3 L-4\right) x^{2}}{4 \Xi(x)^{2}}\right]
\end{aligned}
$$

$$
\begin{aligned}
h_{0,2,0}^{(0)}(\infty)= & \frac{(1-x) x^{2}}{4 \Xi(x)} \\
h_{0,2,0}^{(1)}(\infty)= & -(1-x) x^{2} \\
& \times\left[\frac{1-3 x+(12 L+29) x^{2}-3(4 L+9) x^{3}}{32 \Xi(x)^{5 / 2}}\right. \\
& \left.-x \frac{L+1-L x}{4 \Xi(x)^{2}}\right] \\
h_{0,0,1}^{(0)}(\infty)= & \frac{x(1-x)(3 x-1)}{8 \Xi(x)}
\end{aligned}
$$

$$
\begin{aligned}
h_{0,0,1}^{(1)}(\infty)= & -(1-x) x^{2}\left[(2 L+5) \frac{1-3 x+11 x^{2}-9 x^{3}}{128 \Xi(x)^{5 / 2}}\right. \\
& \left.-x \frac{1+(L-3) x-(L-4) x^{2}}{8 \Xi(x)^{2}}\right] \\
h_{0,3,0}^{(0)}(\infty)= & -\frac{(1-x)^{2} x^{4}}{8 \Xi(x)^{5 / 2}} \\
h_{0,1,1}^{(0)}(\infty)= & -\frac{(1-x)^{2} x^{2}\left(1-2 x+9 x^{2}\right)}{64 \Xi(x)^{5 / 2}}
\end{aligned}
$$

where we have set $\Xi(x)=x(2 x-1)$. 
The ground-state energy per particle is thus given by the following:

$$
\begin{aligned}
e_{0}(N)= & h_{0,0,0}^{(0)}(\infty)+\frac{1}{N} h_{0,0,0}^{(1)}(\infty)+\frac{1}{N^{2}} h_{0,0,0}^{(2)}(\infty) \\
& +\frac{1}{N^{3}} h_{0,0,0}^{(3)}(\infty)+O\left(1 / N^{4}\right),
\end{aligned}
$$

whereas the gap reads

$$
\begin{aligned}
\Delta(N)= & h_{0,1,0}^{(0)}(\infty)+\frac{1}{N} h_{0,1,0}^{(1)}(\infty)+\frac{1}{N^{2}} h_{0,1,0}^{(2)}(\infty) \\
& +O\left(1 / N^{3}\right) .
\end{aligned}
$$

Of course, these expressions coincide for $L=0$ with those given in Refs. $[14,24]$. For $L=2$, one recovers the results given in Ref. [37]. The mean-field result (33) is also recovered in the thermodynamical limit.

It is important to note that the Hamiltonian $H(\infty)=H_{0}(\infty)$ is not diagonal in the eigenbasis of $n_{b}$ (except for $L=0$ ) even though it always commutes with $n_{b}$. Consequently, for each number of excitations, $H$ must be diagonalized.

As can be observed in Eqs. (59)-(71), some divergences appears, for $x=x_{c}$, in the subleading corrections. We will see in Sec. VI that the structure of this singular $1 / N$ expansion at the critical point allows us to extract nontrivial scaling exponents whose determination is one of the main motivation of this work.

\section{Flow equations for $\boldsymbol{b}_{\mu}^{\dagger}$}

We now proceed to derive the flow equation for the operator $b_{\mu}^{\dagger}(l)$ from which any other observable can be obtained. Analogously to the treatment of the Hamiltonian flow equations, the first step consists in transforming the flow equation [Eq. (48)] for $\Omega(l)=b_{\mu}^{\dagger}(l)$ into a set of flow equations for couplings. Therefore, we expand $b_{\mu}^{\dagger}(l)$ in power of $1 / N$. Generically, one may expect to generate any terms $b_{\mu}^{\dagger^{\alpha}} P_{b}^{\dagger^{\beta}}: n_{b}^{\gamma}: P_{b}{ }^{\eta} b_{\mu}{ }^{\nu}$. Here, we shall restrict our discussion to order $1 / N$ for which one only has eight operators

$$
\begin{aligned}
b_{\mu}^{\dagger}(l)= & A_{+}(l) b_{\mu}^{\dagger}+A_{-}(l) \tilde{b}_{\mu}+B_{+}(l) b_{\mu}^{\dagger} n_{b}+B_{-}(l) n_{b} \tilde{b}_{\mu} \\
& +C_{+}(l) P_{b}^{\dagger} \tilde{b}_{\mu}+C_{-}(l) b_{\mu}^{\dagger} P_{b}+D_{+}(l) b_{\mu}^{\dagger} P_{b}^{\dagger} \\
& +D_{-}(l) P_{b} \tilde{b}_{\mu}+O\left(1 / N^{2}\right)
\end{aligned}
$$

where we have introduced $\tilde{b}_{\mu}=(-1)^{\mu} b_{-\mu}$ and where, as previously, each function has a canonical $1 / N$ expansion given by the number of bosonic operators it is associated with, namely

$$
\begin{aligned}
& A_{ \pm}(l)=A^{(0)}{ }_{ \pm}(l)+\frac{A^{(1)}{ }_{ \pm}(l)}{N}+O\left(1 / N^{2}\right), \\
& B_{ \pm}(l)=\frac{B^{(0)}{ }_{ \pm}(l)}{N}+O\left(1 / N^{2}\right), \\
& C_{ \pm}(l)=\frac{C^{(0)}{ }_{ \pm}(l)}{N}+O\left(1 / N^{2}\right), \\
& D_{ \pm}(l)=\frac{D^{(0)}{ }_{ \pm}(l)}{N}+O\left(1 / N^{2}\right) .
\end{aligned}
$$

The initial condition is of course given by: $b_{\mu}^{\dagger}(l=0)=b_{\mu}^{\dagger}$ so that one only has a nonvanishing initial coupling which is $A_{+}^{(0)}(0)=1$. The flow equations are then obtained for these couplings order by order using Eq. (48). The full set of equations is given in Appendix B. As for the couplings defining the running Hamiltonians, these equations can be solved exactly and lead to

$$
\begin{aligned}
A_{s}^{(0)}(\infty) & =\frac{1}{2 \Phi(x)^{1 / 4}} \\
A_{s}^{(1)}(\infty) & =-\frac{(1-x)}{16 x}\left[\frac{2 L+3}{\Phi(x)^{7 / 4}}-\frac{2(L+2)}{\Phi(x)^{5 / 4}}\right] \\
A_{d}^{(0)}(\infty) & =\frac{\Phi(x)^{1 / 4}}{2}, \\
A_{d}^{(1)}(\infty) & =\frac{(1-x)}{16 x}\left[\frac{2 L+3}{\Phi(x)^{5 / 4}}-\frac{2(L+2)}{\Phi(x)^{3 / 4}}\right] \\
B_{s}^{(0)}(\infty) & =-\frac{1-x}{8 x \Phi(x)^{7 / 4}} \\
B_{d}^{(0)}(\infty) & =\frac{1-x}{8 x \Phi(x)^{5 / 4}} \\
C_{s}^{(0)}(\infty) & =-\frac{1-x}{16 x \Phi(x)^{7 / 4}} \\
C_{d}^{(0)}(\infty) & =\frac{1-x}{16 x \Phi(x)^{5 / 4}} \\
D_{s}^{(0)}(\infty) & =\frac{(1-x)(3 x-1)}{32 x^{2} \Phi(x)^{7 / 4}} \\
D_{d}^{(0)}(\infty) & =\frac{1-x^{2}}{32 x^{2} \Phi(x)^{5 / 4}}
\end{aligned}
$$

where we have set $\Phi(x)=(2 x-1) / x, F_{s}=\frac{1}{2}\left(F_{+}+F_{-}\right)$and $F_{d}=\frac{1}{2}\left(F_{+}-F_{-}\right)$, for each function $F=A, B, C, D$.

The above expansion of $b_{\mu}^{\dagger}(\infty)$ allows us to compute $\left\langle\Psi|\Omega| \Psi^{\prime}\right\rangle$ for any operator $\Omega$ that can be expressed in terms of $b_{\mu}^{\dagger}$ and for any eigenstates $|\Psi\rangle$ and $\left|\Psi^{\prime}\right\rangle$ of the Hamiltonian $H(\infty)$. In the following, we consider two different examples to show the power of this approach.

\section{Expectation value of the occupation number $n_{L}$}

Let us first consider the case where $\Omega=n_{L}$ and where $|\Psi\rangle=\left|\Psi^{\prime}\right\rangle$ is the ground state of $H$. This quantity normalized by the number of bosons can be computed straightforwardly by means of the Hellmann-Feynman theorem which states

$$
\frac{\left\langle n_{L}\right\rangle}{N}=\frac{\partial}{\partial y}\left[(1+y) h_{0,0,0}\right],
$$

where $y=x /(1-x)$. Because we have the expansion of $h_{0,0,0}$ up to order $(1 / N)^{3}$, one can easily get $\left\langle n_{L}\right\rangle$ at this order. Here, instead, we compute it in terms of the flow equation for the operator $b_{\mu}^{\dagger}$ obtained in the preceding section, using the fact that:

$$
n_{L}(\infty)=n_{b}(\infty)=\sum_{\mu} b_{\mu}^{\dagger}(\infty) b_{\mu}(\infty)
$$


where $b_{\mu}^{\dagger}(\infty)$ is given by Eq. (74) with final values Eqs. (79)(88). The ground state of the Hamiltonian (57) being defined as the zero $b$ boson state $|0\rangle$, one has the following:

$$
\begin{aligned}
\frac{\left\langle 0\left|n_{L}(\infty)\right| 0\right\rangle}{N} \\
=\frac{1}{N} \sum_{\mu}\left\langle 0\left|b_{\mu}^{\dagger}(\infty) b_{\mu}(\infty)\right| 0\right\rangle \\
=\frac{1}{N} \sum_{\mu} A_{-}^{2}(\infty)\left\langle 0\left|b_{\mu} b_{\mu}^{\dagger}\right| 0\right\rangle+O\left(1 / N^{3}\right), \\
=\frac{1}{N}(2 L+1)\left[A_{-}^{(0)}(\infty)^{2}+\frac{2}{N} A_{-}^{(0)}(\infty) A_{-}^{(1)}(\infty)\right]+O\left(1 / N^{3}\right), \\
=\frac{(2 L+1)}{N}\left[\frac{3 x-1}{4 \Xi(x)^{1 / 2}}-\frac{1}{2}\right]+\frac{(2 L+1)}{N^{2}} \frac{x(1-x)^{2}}{16} \\
\quad \times\left[-\frac{(2 L+3) x}{\Xi(x)^{2}}+\frac{2(L+2)}{\Xi(x)^{3 / 2}}\right]+O\left(1 / N^{3}\right),
\end{aligned}
$$

where, as previously, $\Xi(x)=x(2 x-1)$. It can be easily verified that this expression coincides with Eq. (89).

\section{E. Transition probability between the ground state and the first excited state}

As explained above, the real power of the CUTs method is that it allows to easily compute off-diagonal matrix elements of any operator between any eigenstates of the Hamiltonian provided one knows the expression of the associated running operator. As an example, we focus here on the transition probability $T=\left|\left\langle 1\left|T_{L_{0}}\right| 0\right\rangle\right|^{2}$ between the ground state $|0\rangle$ and the first excited state $|1\rangle$. The operator $T_{L_{\mu}}$ was defined in Eq. (24). It is important to note that here the ground state has a zero angular momentum, whereas the first excited state has an angular momentum $L$. To determine the matrix element of $T_{L_{0}}$ of interest, we shall proceed as for the occupation number and consider its $1 / N$ expansion in terms of the $b$ boson:

$$
\begin{aligned}
T_{L_{0}} & =s^{\dagger} L_{0}+L_{0}^{\dagger} s \\
& =N^{1 / 2}\left[b_{0}^{\dagger}+b_{0}-\frac{1}{2 N}\left(b_{0}^{\dagger} n_{b}+n_{b} b_{0}\right)+O\left(1 / N^{2}\right)\right] .
\end{aligned}
$$

To be consistent, given that we only have the expression of $b_{0}^{\dagger}(\infty)+b_{0}(\infty)$ at order $1 / N$, we need to consider $b_{0}^{\dagger}(\infty) n_{b}(\infty)+n_{b}(\infty) b_{0}(\infty)$ at order $(1 / N)^{0}$. Using the expression (74) of the operator $b_{0}^{\dagger}$, one easily gets

$$
\left\langle 1\left|b_{0}^{\dagger}(\infty)+b_{0}(\infty)\right| 0\right\rangle=A_{+}(\infty)+A_{-}(\infty),
$$

and

$$
\begin{aligned}
\left\langle 1\left|b_{0}^{\dagger}(\infty) n_{b}(\infty)+n_{b}(\infty) b_{0}(\infty)\right| 0\right\rangle \\
=(2 L+3) A_{+}(\infty) A_{-}(\infty)^{2}+A_{-}(\infty) \\
\quad \times\left[(2 L+2) A_{-}(\infty)^{2}+A_{+}(\infty)^{2}\right] .
\end{aligned}
$$

Truncating these expressions at order $1 / N$ and $(1 / N)^{0}$ respectively and using Eqs. (75), (79) and (80), one finally obtains the following:

$$
\begin{aligned}
\frac{T}{N}= & \frac{x}{\Xi(x)^{1 / 2}}+\frac{x^{2}}{N} \\
& \times\left[-\frac{(2 L+1)-4(2 L+1) x+(10 L+7) x^{2}}{4 \Xi(x)^{2}}\right. \\
& \left.+\frac{-L+(3 L+2) x}{2 \Xi(x)^{3 / 2}}\right]+O\left(1 / N^{2}\right)
\end{aligned}
$$

As for the expansion of the spectrum, some singularities appears, and we shall see that they also provide the scaling exponents at the critical point.

\section{THE BROKEN PHASE $(0<x<1 / 2)$}

As shown by the mean-field analysis, for $x<1 / 2$, the order parameter $\left\langle n_{L}\right\rangle / N$ has a nonvanishing value. This implies that we have to consider a new vacuum for the Holstein-Primakoff expansion. Therefore, we shift the bosonic modes by a term proportional to $\sqrt{N}$. We thus define the $c$ bosons by the following:

$$
b_{\mu}^{\dagger}=\sqrt{N} \lambda_{\mu}^{*}+c_{\mu}^{\dagger}
$$

where the $\lambda_{\mu}$ 's are complex numbers that form a $(2 L+1)$ dimensional vector. Of course, the symmetric phase results are recovered when setting $\lambda_{\mu}=0$. Then, using Eqs. (36)-(38) and assuming that $n_{c} / N \ll 1$, we expand the Hamiltonian that now contains some a term proportional to $\sqrt{N}$ that reads as follows:

$$
\begin{aligned}
& \left(c^{\dagger} \cdot \tilde{\lambda}+\lambda^{\dagger} \cdot \tilde{c}\right)\left\{x+\frac{1-x}{4}\left[-2\left(1-n_{\lambda}\right)+P_{\lambda}^{\dagger}+P_{\lambda}\right]\right\} \\
& +\frac{1-x}{2}\left[\left(c^{\dagger} \cdot \lambda^{\dagger}\right)\left(P_{\lambda}-1+n_{\lambda}\right)+(\lambda \cdot c)\left(P_{\lambda}^{\dagger}-1+n_{\lambda}\right)\right],
\end{aligned}
$$

where $\lambda_{\mu}^{\dagger}=\lambda_{\mu}^{*}$ and $\tilde{\lambda}_{\mu}=(-1)^{\mu} \lambda_{-\mu}$. There are several choices of the $\lambda_{\mu}$ s that allow one to get rid of these terms. Here, we have chosen to set $\lambda_{\mu}=\lambda_{0} \delta_{\mu, 0}$ with $\lambda_{0}^{2}=(1-2 x) /[2(1-x)]$. Note that in the thermodynamical limit, we recover the mean-field value [Eq. (35)]

$$
\frac{\left\langle n_{L}\right\rangle}{N}=\sum_{\mu}\left|\lambda_{\mu}\right|^{2}=\frac{1-2 x}{2(1-x)} .
$$

Further, we emphasize that this choice of the $\lambda_{\mu} \mathrm{s}$ is the same as the one proposed in the mean-field analysis where we have broken the spherical symmetry by populating macroscopically $\mu=0$ boson level only. With this choice, the Hamiltonian reads as follows:

$$
\begin{aligned}
H= & -N x \frac{3 x-2}{4(1-x)}+N^{0}\left[\frac{(1-3 x)(1-2 x)}{8(1-x)}+\frac{x}{2} n_{c}\right. \\
& \left.+\frac{5}{4}(1-2 x) c_{0}^{\dagger} c_{0}-\frac{x}{4}\left(P_{c}^{\dagger}+P_{c}\right)+\frac{3}{8}(1-2 x)\left(c_{0}^{\dagger^{2}}+c_{0}^{2}\right)\right] \\
& +O(1 / \sqrt{N}) .
\end{aligned}
$$

Contrary to the symmetric phase, we do restrict our discussion to this order because, as shown later, the existence of gapless modes at this level does not allow us to go beyond this order with the CUTs. 


\section{A. The spectrum}

The Hamiltonian (99) can be easily diagonalized via a Bogoliubov transform. Therefore, we introduce the $d$ bosons defined by the following:

$$
\begin{aligned}
& c_{\mu}^{\dagger}=\cosh \left(\Theta_{\mu} / 2\right) d_{\mu}^{\dagger}+\sinh \left(\Theta_{\mu} / 2\right) \tilde{d}_{\mu}, \\
& \tilde{c}_{\mu}=\sinh \left(\Theta_{\mu} / 2\right) d_{\mu}^{\dagger}+\cosh \left(\Theta_{\mu} / 2\right) \tilde{d}_{\mu} .
\end{aligned}
$$

The angles $\Theta_{\mu}$ are chosen so that $H$ written in terms of the $d$ 's is diagonal. From Eq. (99), it is clear that modes with $\mu \neq 0$ and $\mu=0$ plays a different role and actually decouple. As can be easily seen, eliminating off-diagonal terms for $\mu \neq 0$ implies to set $\Theta_{\mu \neq 0}=\infty$ and gives $2 L$ gapless modes. Because such a transform is singular, one has to use another route. The contribution of terms with $\mu \neq 0$ in the Hamiltonian reads as follows:

$$
H_{\mu}+H_{-\mu}=\frac{x}{2}\left[c_{\mu}^{\dagger} c_{\mu}+c_{-\mu}^{\dagger} c_{-\mu}-(-1)^{\mu}\left(c_{\mu}^{\dagger} c_{-\mu}^{\dagger}+c_{-\mu} c_{\mu}\right)\right] .
$$

Introducing the position and momentum operator

$$
X_{\mu}=\frac{c_{\mu}^{\dagger}+c_{\mu}}{\sqrt{2}}, \quad P_{\mu}=i \frac{c_{\mu}^{\dagger}-c_{\mu}}{\sqrt{2}},
$$

one has

$$
\begin{aligned}
H_{\mu}+H_{-\mu}= & -\frac{x}{2}+\frac{x}{4}\left[\left(P_{\mu}+(-1)^{\mu} P_{-\mu}\right)^{2}\right. \\
& \left.+\left(X_{\mu}-(-1)^{\mu} X_{-\mu}\right)^{2}\right] .
\end{aligned}
$$

Because $\left[P_{\mu}+(-1)^{\mu} P_{-\mu}, X_{\mu}-(-1)^{\mu} X_{-\mu}\right]=0, H_{\mu}$ is written in a diagonal form and its spectrum is indeed found to be gapless and continuous. The correction to the ground-state energy coming from this contribution is thus $-L x / 2$.

Let us now consider the $\mu=0$ part of the Hamiltonian that reads:

$$
H_{\mu=0}=\left(\frac{5}{4}-2 x\right) c_{0}^{\dagger} c_{0}+\left(\frac{3}{8}-x\right)\left(c_{0}^{\dagger^{2}}+c_{0}^{2}\right) .
$$

For this contribution, the Bogoliubov transform can be simply achieved and one obtains the following:

$$
H_{\mu=0}=\frac{1}{2}\left(\sqrt{1-2 x}-\frac{5}{4}+2 x\right)+\sqrt{1-2 x} d_{0}^{\dagger} d_{0} .
$$

The correction to the ground-state energy coming from this contribution is thus given by the $d_{0}$ boson state. As a result, $e_{0}$ at this order reads as follows:

$$
\begin{aligned}
e_{0}(N)= & \frac{x}{4} \frac{2-3 x}{1-x}+\frac{1}{N}\left[\frac{-2-2(L-2) x+(2 L-1) x^{2}}{4(1-x)}\right. \\
& \left.+\frac{1}{2} \sqrt{1-2 x}\right]+O\left(1 / N^{2}\right) .
\end{aligned}
$$

As in the symmetric phase, the leading corrections coincide with the mean-field result (34) and $L$ only appears in the subleading terms.

Concerning the gap, the above analysis indicates the existence of $2 L$ gapless modes and a gapped one with excitation energy

$$
\Delta^{\prime}(N)=\sqrt{1-2 x}+O(1 / N) .
$$

As previously, one can simply obtain $\left\langle n_{L}\right\rangle / N$ by replacing $h_{0,0,0}$ by $e_{0}(N)$ in Eq. (89), and the result is as follows:

$$
\begin{aligned}
\frac{\left\langle n_{L}\right\rangle}{N}= & \frac{1-2 x}{2(1-x)}-\frac{1}{2 N} \\
& \times\left[L+\frac{x}{\sqrt{1-2 x}}+\frac{x}{x-1}\right]+O\left(1 / N^{2}\right) .
\end{aligned}
$$

At this stage, one can understand the difficulty to go beyond this order in the presence of gapless modes. Indeed, computing the next-order corrections would imply to keep on making the $1 / N$ expansion around the (broken) vacuum, but such a procedure does not take into account the degeneracy because of the gapless modes. Note that for $L=0$ where no gapless modes emerge, we have been able to compute these corrections using CUTs [24]. To conclude this subsection, we wish to underline that in the two-level BCS model where gapless modes also exist, Richardson has obtained the finite-size corrections in the broken phase beyond the Bogoliubov order using the $1 / N$ expansion of the exact solution [38], whereas we computed them more recently using CUTs in the symmetric phase [39].

\section{B. Transition probability between the ground state and the first excited state}

As in the symmetric phase, we now compute the transition $T=\left|\left\langle 1\left|T_{L_{0}}\right| 0\right\rangle\right|^{2}$, where $T_{L_{0}}=s^{\dagger} L_{0}+L_{0}^{\dagger} s$. However, the important difference is that, in the broken phase, one has $2 L$ gapless modes that renders the definition of the first excited states more tricky. In the thermodynamical limit, the ground state thus becomes infinitely degenerate and one actually has to simply consider the expectation value of $T_{L_{0}}$ over the ground state. To avoid any confusion, we call this quantity $T^{\prime}$ instead of $T$. Using the expansion (93) and the shift (96) with the choice of the $\lambda_{\mu}$ given previously, one obtains the following:

$$
\begin{aligned}
T^{\prime} & =\left|\left\langle 0\left|T_{L_{0}}\right| 0\right\rangle\right|^{2}, \\
& =N^{2} 4 \lambda_{0}^{2}\left(1-\lambda_{0}^{2}\right)+O(N), \\
& =N^{2} \frac{1-2 x}{(1-x)^{2}}+O(N) .
\end{aligned}
$$

First, it is important to note that $T^{\prime}$ is proportional to $N^{2}$ in this phase, whereas $T$ scales as $N$ in the symmetric phase. Second, in the broken phase, $T^{\prime}$ vanishes at the critical point, whereas $T$ diverges when approaching from the symmetric phase. This result clearly suggests an anomalous scaling behavior at the critical point that we shall now investigate in details.

\section{THE CRITICAL POINT}

In this section, we analyze the behavior of the $1 / N$ expansion of the quantities considered in this study: the ground-state energy, the gap, the expectation value of $n_{L}$ in the ground state, and the transition rate $T$ between the ground state and the first excited state. The common point of all these expansions is that they become singular at the critical point. Following the arguments presented in a recent series 
of papers $[14,24,37,39]$ we now recall how this intriguing property allows one to extract the finite-size scaling exponents at this point.

All quantities considered in this study display a singular behavior for $x=x_{c}$. This singular behavior can emerge in subleading corrections as for the ground-state energy but also in the leading term as illustrated by the transition rate in the symmetric phase [see Eq. (95)]. Thus, schematically, the $1 / N$ expansion of a physical quantity $\Phi$ can be decomposed into a regular and a singular part as

$$
\Phi_{N}(x)=\Phi_{N}^{\mathrm{reg}}(x)+\Phi_{N}^{\mathrm{sing}}(x),
$$

where, contrary to $\Phi_{N}^{\text {sing }}, \Phi_{N}^{\text {reg }}$ and all its derivatives with respect to $x$ do not diverge when $x$ goes to $x_{c}$. Furthermore, at each order of the expansion, the divergence of $\Phi_{N}^{\mathrm{sing}}$ is dominated by a single term. To be more concrete, let us consider the ground-state energy in the symmetric phase for which

$$
\begin{aligned}
\Phi_{N}^{\mathrm{reg}}(x)= & \frac{1-x}{4}+\frac{1}{N} \frac{(2 L+1)(1-3 x)}{4}, \\
\Phi_{N}^{\mathrm{sing}}(x)= & \frac{1}{N} \frac{(2 L+1) \Xi(x)^{1 / 2}}{2}+\frac{1}{N^{2}} h_{0,0,0}^{(2)}(\infty) \\
& +\frac{1}{N^{3}} h_{0,0,0}^{(3)}(\infty)+O\left(1 / N^{4}\right) .
\end{aligned}
$$

In the vicinity of the critical point, these diverging terms have a leading contribution that is proportional to $\Xi(x)^{-1}$ for $h_{0,0,0}^{(2)}(\infty)$ and to $\Xi(x)^{-5 / 2}$ for $h_{0,0,0}^{(3)}(\infty)$. This has lead us to conjecture that near $x_{c}$ the singular part behaves as follows:

$$
\Phi_{N}^{\text {sing }}(x) \simeq \frac{\Xi(x)^{\xi_{\Phi}}}{N^{n_{\Phi}}} \mathcal{F}_{\Phi}\left[N \Xi(x)^{3 / 2}\right]
$$

where $\mathcal{F}_{\Phi}$ is a function that only depends on the scaling variable $N \Xi(x)^{3 / 2}$. We underline that for the LMG model $(L=0)$ we have checked this scaling hypothesis up to a high order in the $1 / N$ expansion. For the ground-state energy discussed above, one has $\xi_{\Phi}=1 / 2$ and $n_{\Phi}=1$.

Once the form [Eq. (114)] is accepted, the scaling exponents are directly obtained. Indeed, because at finite $N$, physical quantities must not diverge, we conclude that, necessarily, $\mathcal{F}_{\Phi}(x) \sim x^{-2 \xi_{\Phi} / 3}$ so that finally one has, $\Phi_{N}^{\text {sing }}\left(x_{\mathrm{c}}\right) \sim N^{-\left(n_{\Phi}+2 \xi_{\Phi} / 3\right)}$. In Table I we have gathered the exponents obtained for the quantities discussed in this article.

We wish to emphasize that the scaling exponents related to the spectral quantities, i.e., $e_{0}, \Delta$ and, using Eq. (89), $\left\langle n_{L}\right\rangle$, can also be obtained in a different way. Indeed, as explained in Sec. III, the energy surface is the one of a quartic oscillator

TABLE I. Scaling exponents for the ground-state energy per boson $e_{0}$, the gap $\Delta$, the number of $L$ bosons in the ground state $\left\langle n_{L}\right\rangle$, and the $T$ transition probability.

\begin{tabular}{lcrc}
\hline \hline$\Phi$ & $\xi_{\Phi}$ & $n_{\Phi}$ & $-\left(n_{\Phi}+2 \xi_{\Phi} / 3\right)$ \\
\hline$e_{0}$ & $1 / 2$ & 1 & $-4 / 3$ \\
$\Delta$ & $1 / 2$ & 0 & $-1 / 3$ \\
$\left\langle n_{L}\right\rangle$ & $-1 / 2$ & 0 & $1 / 3$ \\
$T$ & $-1 / 2$ & -1 & $4 / 3$ \\
\hline \hline
\end{tabular}

( $\beta^{4}$-like potential) and this can be used as a starting point of a semiclassical description to show that the spectrum, at the critical point scales as $N^{-1 / 3}$. For technical details, we refer the reader to Ref. [13] for the LMG model or [40] for the IBM with $L=2$, and we also note that the "critical" scaling exponents do not depend on $L$. However, the present approach has a real advantage as compared to this latter method because one can compute the scaling exponents of any observables using the expression of $b_{\mu}(\infty)$. We are further not restricted to expectation value but we can also investigate off-diagonal terms as illustrated with the transition rate $T$.

\section{NUMERICAL RESULTS}

In this section we check the validity of the analytical expressions obtained in the preceding sections using CUTs. The observables studied are the ground-state energy per boson $e_{0}$, the gap $\Delta$, the expectation value of the number of $L$ bosons in the ground state $\left\langle n_{L}\right\rangle$, and the transition probability between the ground state and the first excited state $T$.

In Fig. 4 we present the general features of the selected observables as a function of the control parameter $x$ for $L=2$ and for $N=500$. Note that in the broken phase $\left(x<x_{c}\right)$, we have plotted $\Delta$ and $T / N^{2}$ instead of $\Delta^{\prime}$ and $T^{\prime} / N^{2}$, these two latter quantities are discussed under Sec. VII B. We can thus clearly appreciate the emergence of Goldstone modes in the broken phase. We emphasize that $\left\langle n_{L}\right\rangle / N$ as well as the transition probability $T / N^{2}$ may be considered order parameters because they vanish in the symmetric phase and acquire a finite value in the broken one. However, although $\left\langle n_{L}\right\rangle / N$ is directly related to the physical ground state, $T$ involves the first excited state that turns out to collapse into the ground state in the broken phase. This latter property makes it a more controversial candidate for an order parameter as recently discussed in Refs. [19,41].

In Fig. 5, we plot the difference between the numerical and the mean-field value of $e_{0}$ (dashed line) and $\left\langle n_{L}\right\rangle / N$ (full line) as a function of the boson number, $N$, for three characteristic
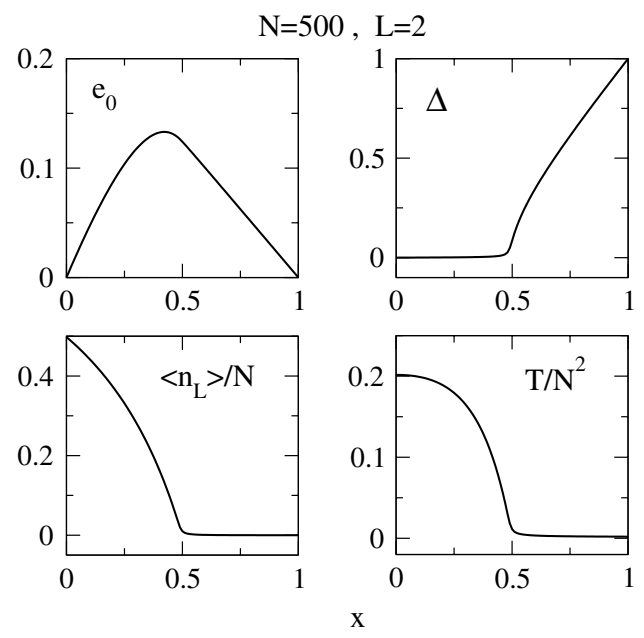

FIG. 4. General features of the observables studied in this work as a function of the control parameter $x$ obtained by numerical diagonalization. 


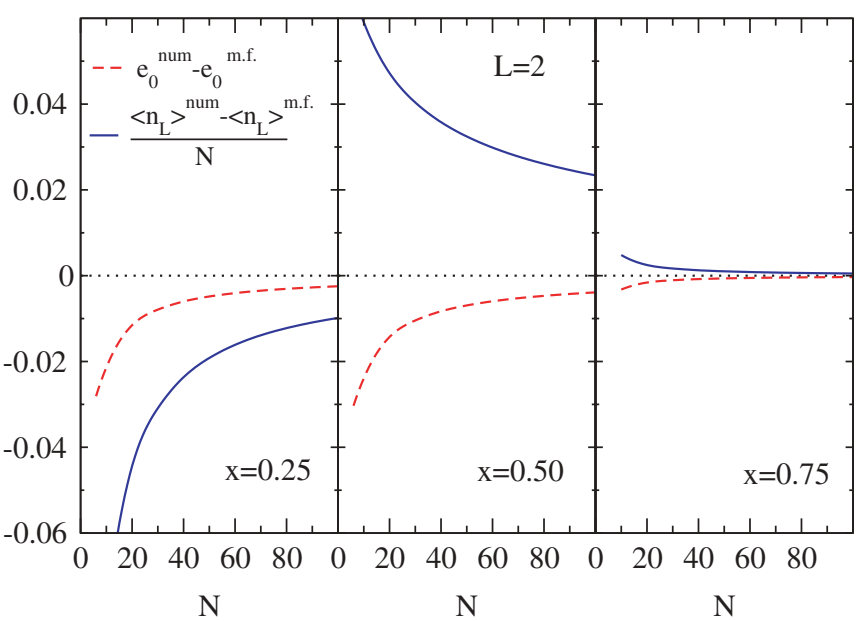

FIG. 5. (Color online) Differences between numerical (num) and mean-field (m.f.) results for the ground-state energy (per boson) $e_{0}$ and expectation value of the number of $L=2$ bosons in the ground state (per boson) $\left\langle n_{L}\right\rangle / N$ as a function of the boson number $N$ for three values of the control parameter as follows: $x=0.25$ (broken phase), $x=0.5$ (critical point), and $x=0.75$ (spherical phase).

values of the control parameter as follows: $x=0.75$ in the symmetric phase, $x=0.5$ at the critical point, and $x=0.25$ in the broken phase. As can be seen, the mean-field results become exact when increasing the number of bosons. It is interesting to note the change in sign in the deviations of the order parameter showing that the mean-field approach underestimates (overestimates) it in the symmetric phase (in the broken phase). To emphasize this effect, we plot in Fig. 6 the same quantities for $N=20$ bosons as a function of the control parameter $x$. Although deviations in the ground-state energy behave smoothly around the critical point, there is a well-defined cusp in the deviations of the $\left\langle n_{L}\right\rangle / N$.

Now that we have shown the main characteristics of the physical quantities of interest and the general agreement, in the thermodynamical limit, with the simple mean-field results

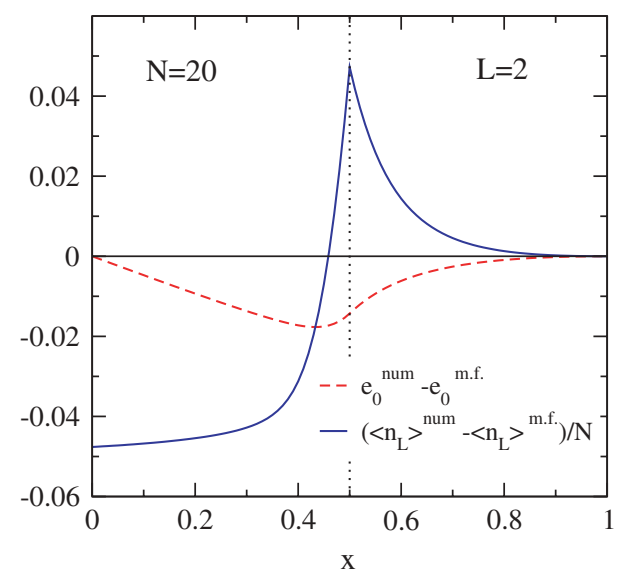

FIG. 6. (Color online) Differences between numerical (num) and mean-field (m.f.) results for the ground-state energy (per boson) $e_{0}$ and expectation value of the number of $L=2$ bosons in the ground state (per boson) $\left\langle n_{L}\right\rangle / N$ as a function of the control parameter $x$ at fixed $N=20$.

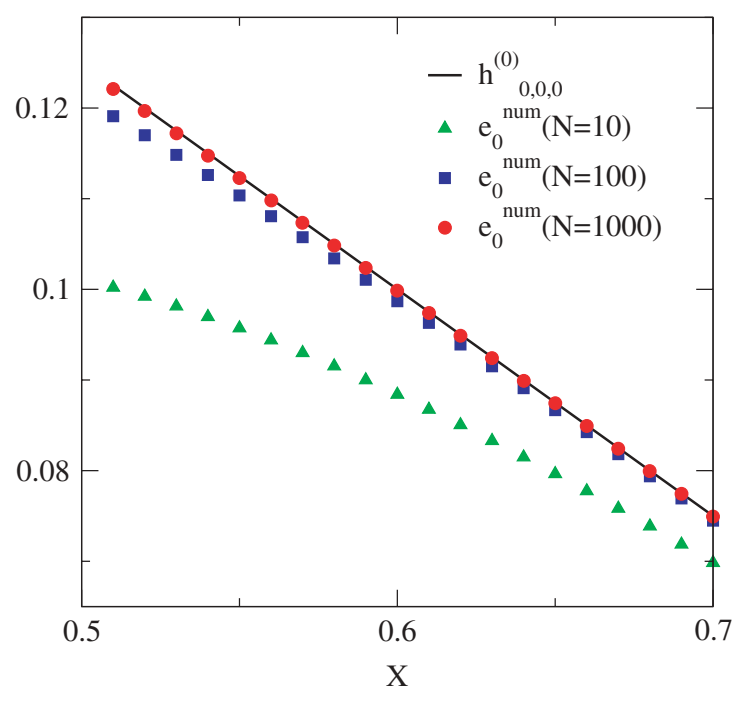

FIG. 7. (Color online) Comparison between the numerical (symbols) and analytical (line) ground-state energy per boson $e_{0}$ for different values of $N$ at leading order.

presented in Sec. III, let us analyze in details the finite-size corrections in each phase independently.

\section{A. The symmetric phase}

In Sec. IV, we have obtained analytical expressions for the different corrections in the $1 / N$ expansion of the selected observables. To check these results, we present several plots focusing in a first step, on the case $L=2$ and the dependence with $x$, whereas, in a second step, we discuss the dependence with $L$.

Let us first consider the ground-state energy per boson $e_{0}$ whose expansion in the symmetric phase is given in Eq. (72). In Fig. 7, the leading term in Eq. (72) is compared with the numerical results for different $N$ values confirming that the $h_{0,0,0}^{(0)}$ is indeed the true asymptotic value of $e_{0}$.

Next, we compare in Fig. 8 the numerical and analytical subleading corrections to $e_{0}$ at each order. The numerical

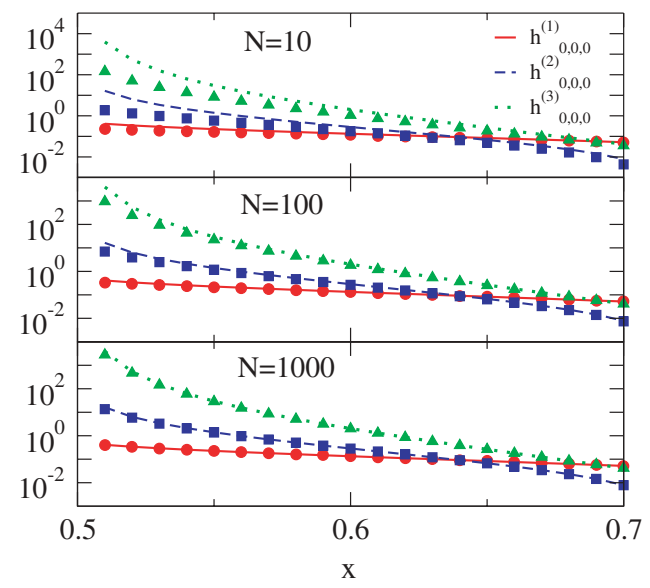

FIG. 8. (Color online) Comparison in log-normal scale between the numerical (symbols) and analytical results (lines) order by order for the ground-state energy per boson $e_{0}$ (see text for definitions). 


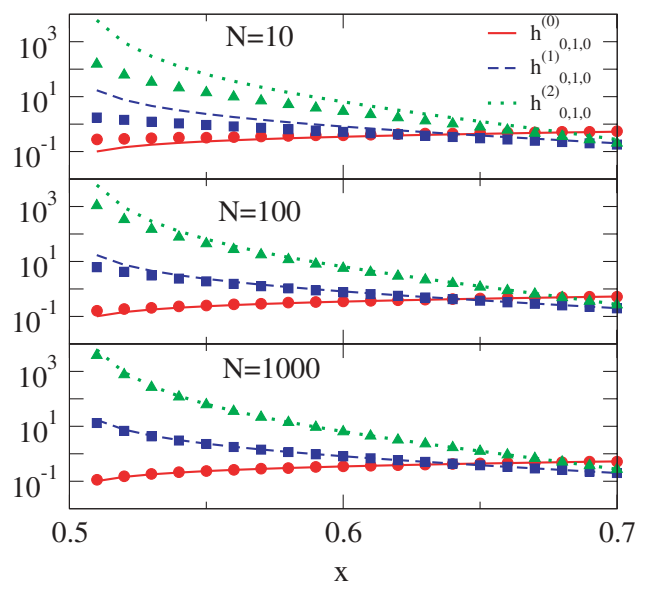

FIG. 9. (Color online) Comparison in log-normal scale between the numerical (symbols) and analytical results (lines) order by order for the gap $\Delta$ (see text for definitions).

corrections of order $p$ to $e_{0}$ are defined from the numerical value $e_{0}^{\text {num }}$ as $N^{p}\left|e_{0}^{\text {num }}-\sum_{\alpha=0}^{p-1} h_{0,0,0}^{(\alpha)}(\infty) / N^{\alpha}\right|$, whereas the analytical correction is obviously given by $h_{0,0,0}^{(p)}(\infty)$. We present results for $p=1,2,3$ and $N=10,100,1000$. As can be clearly seen, for the largest value of $N=1000$, numerical and analytical results are almost indistinguishable even for values of $x$ close to the critical point where $h_{0,0,0}^{(2)}$ and $h_{0,0,0}^{(3)}$ are known to diverge. Note that the critical point $x_{c}=0.5$ was explicitly excluded. Of course, the smaller $N$ the larger the discrepancy because the numerical correction defined above still contains higher-order terms which play a role in this case.

Along the same line, we analyze in Fig. 9 the corrections for the gap $\Delta$ defining the numerical correction of order $p$ from the numerically calculated gap $\Delta^{\text {num }}$ as $N^{p} \mid \Delta^{\text {num }}-$ $\sum_{\alpha=-1}^{p-1} h_{0,1,0}^{(\alpha)}(\infty) / N^{\alpha} \mid$ with $h_{0,1,0}^{(-1)}(\infty)=0$. The analytical correction of order $p$ is $h_{0,1,0}^{(p)}(\infty)$.

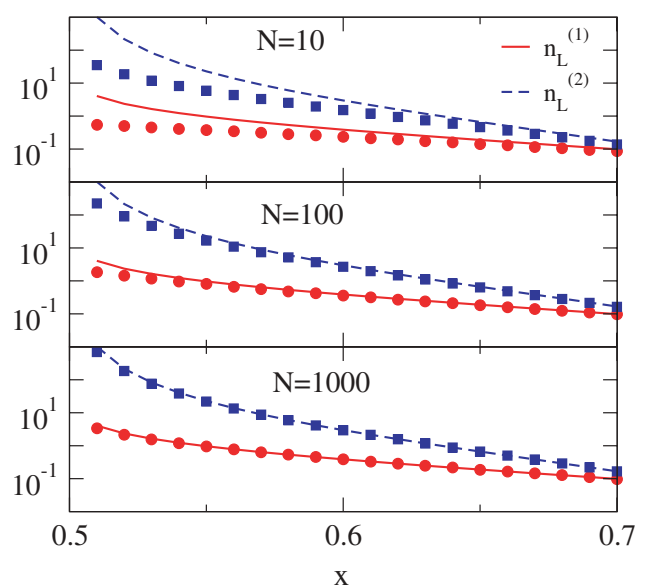

FIG. 10. (Color online) Comparison in log-normal scale between the numerical (symbols) and analytical results (lines) for the expectation value of the occupation number in the $L$ level per boson in the ground state $\left\langle n_{L}\right\rangle / N . n_{L}^{(1)}$ stands for the $1 / N$ term and $n_{L}^{(2)}$ stands for the $1 / N^{2}$ term in Eq. (91).

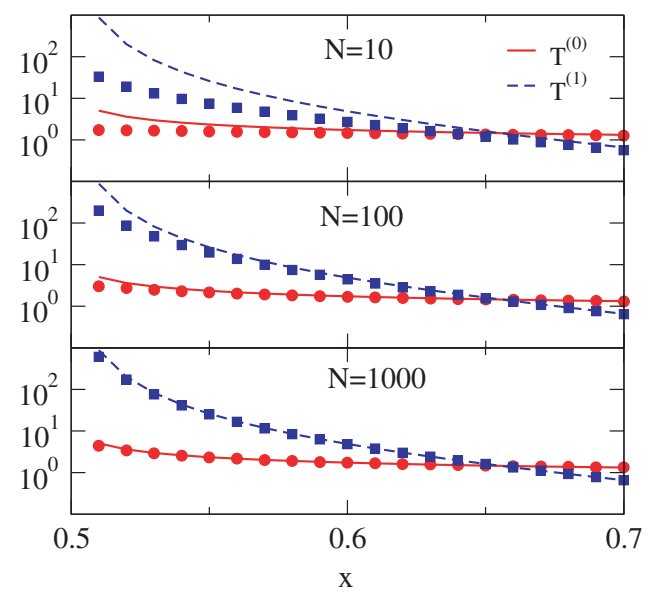

FIG. 11. (Color online) Comparison in log-normal scale between the numerical (symbols) and analytical results (lines) for the transition probability per boson between the ground and the first excited state $T / N . T^{(0)}$ stands for the $N$-independent term and $T^{(1)}$ stands for the $1 / N$ term in Eq. (95).

Finally, we perform the same analysis for $\left\langle n_{L}\right\rangle / N$ (see Fig. 10) and $T / N$ (see Fig. 11) by comparing the two first terms of their expansion with the numerical results. The numerical corrections are computed as for the gap. All these plots reflect that the $x$ dependence of the analytical expressions obtained with CUTs are in complete agreement with the exact numerical results for large values of $N$.

To end up with these checks, we have investigated the $L$ dependence of the analytical results. We present in Fig. 12 the same observables (with the same notations) as those presented in Figs. 8-11 for fixed $x=0.6$ and $N=1000$ as a function of $L$. Once again, the agreement between the numerical results and the analytical expressions is excellent and confirms the validity of our analytical results.

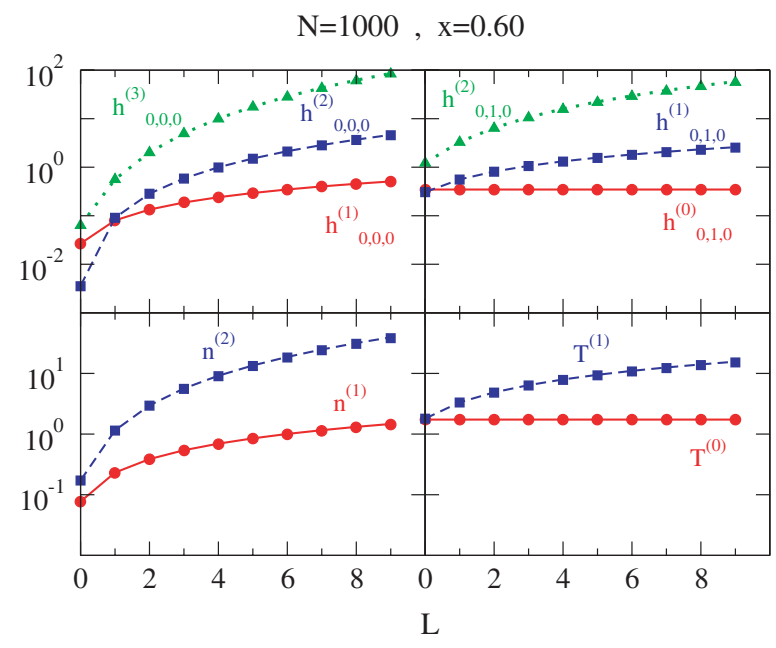

FIG. 12. (Color online) Comparison in log-normal scale between numerical (symbols) and analytical results (lines) as a function of $L$. Notations are the same as in Figs. 8-11. 

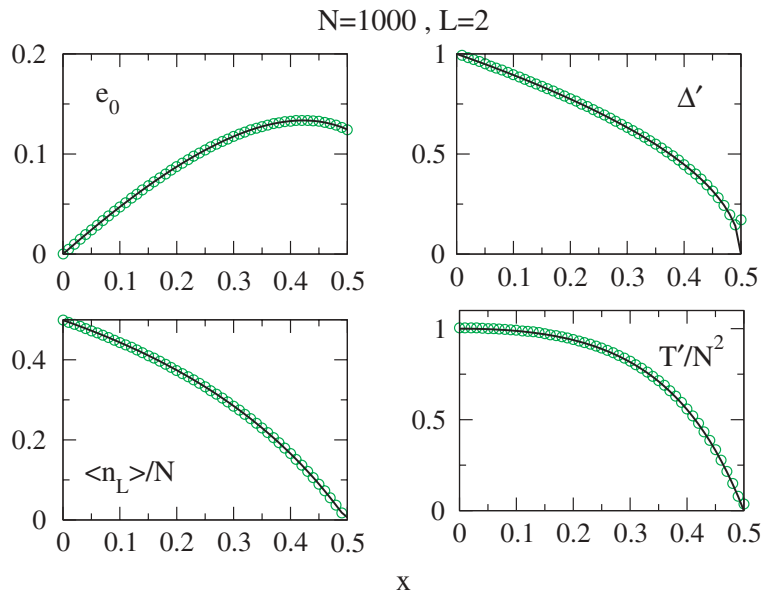

FIG. 13. (Color online) Comparison between numerical (symbols) and analytical (lines) results. We plot here only the leading terms for each quantity.

\section{B. The broken phase}

As explained in Sec. V, the presence of Goldstone modes in the broken phase prevents computation of the corrections at a high order. Therefore, we restrict our discussion here to the first nontrivial order. We present in Fig. 13 a direct comparison between numerical results (symbols) and the analytical ones given in Eqs. (107), (108), (109), and (110) (lines) as a function of the control parameter $x$ for $N=1000$ and $L=2$.

It is worth reminding the reader that the gap associated with a one-phonon state in the symmetric phase turns into a Goldstone boson in the broken phase. The first excited state in the latter phase thus corresponds to a two-phonon state in the symmetric phase. However, this gapped mode [Eq. (108)] goes to zero at the critical point in the thermodynamic limit $N=\infty$. As in the symmetric case, one can appreciate the agreement between analytics and numerics as already discussed, at this order, in Ref. [21].

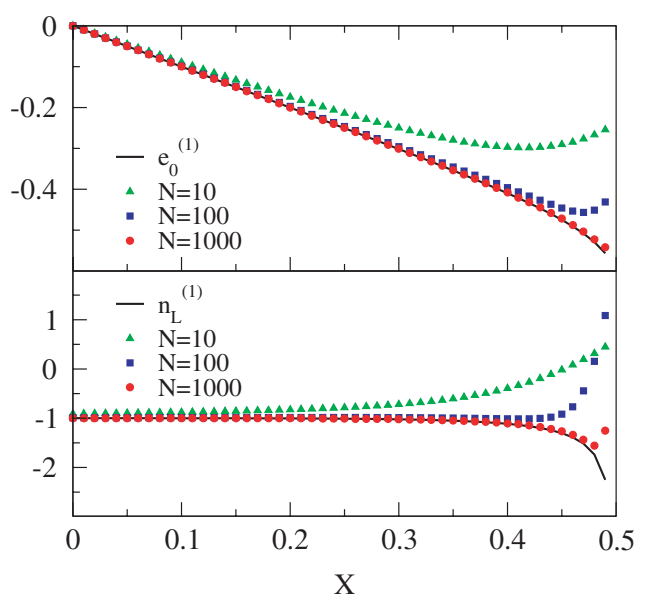

FIG. 14. (Color online) Comparison between numerical (symbols) and analytical (lines) results for $L=2$. As in the symmetric phase, we have substracted from the numerical data the leading term given by the mean-field treatment.

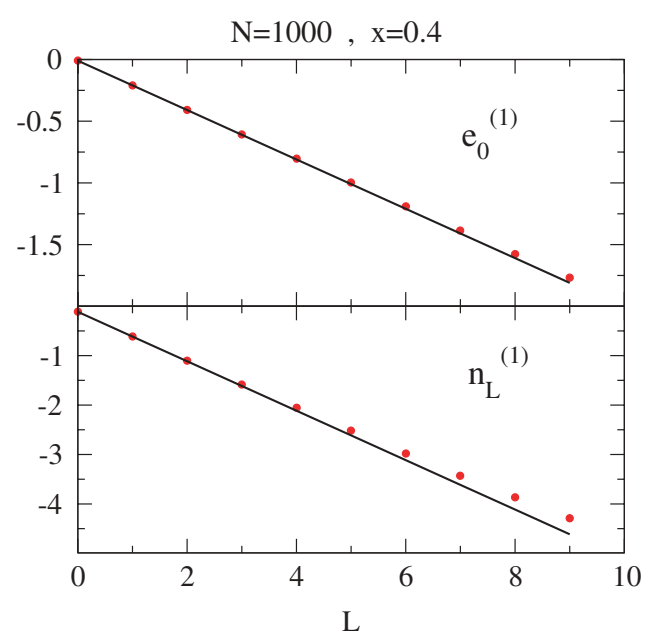

FIG. 15. (Color online) Comparison between numerical (symbols) and analytical (lines) results for the subleading corrections. As in the symmetric phase, we have substracted from the numerical data the leading term; $e_{0}^{(1)}$ and $n_{L}^{(1)}$ refers respectively to the term proportional to $1 / N$ in Eq. (107) and Eq. (109) respectively.

We have also checked that the subleading terms of $e_{0}$ and $\left\langle n_{L}\right\rangle / N$ (beyond the mean-field results), which contains a nontrivial dependence with $L$, were fitting with numerics. In Fig. 14, we show for $L=2$, a comparison between numerical and analytical results for $N=10,100,1000$. As in the symmetric phase, the larger $N$ the better the agreement. The dependence with $L$ is tested in Fig. 15 at fixed $x$ and $N$.

\section{The critical point}

We now turn to the critical point study. To check the value of the finite-size scaling exponents derived in Sec. VI, we have performed diagonalizations for large system size (up to $N=2^{13}$ bosons). Let us recall that for $L=0$, we have checked these values for larger system size in Ref. [24]. We show in Fig. 16 our results for different values of $L=0,1,2,3$. Note that we plot the $\log _{2}$ of each quantity.

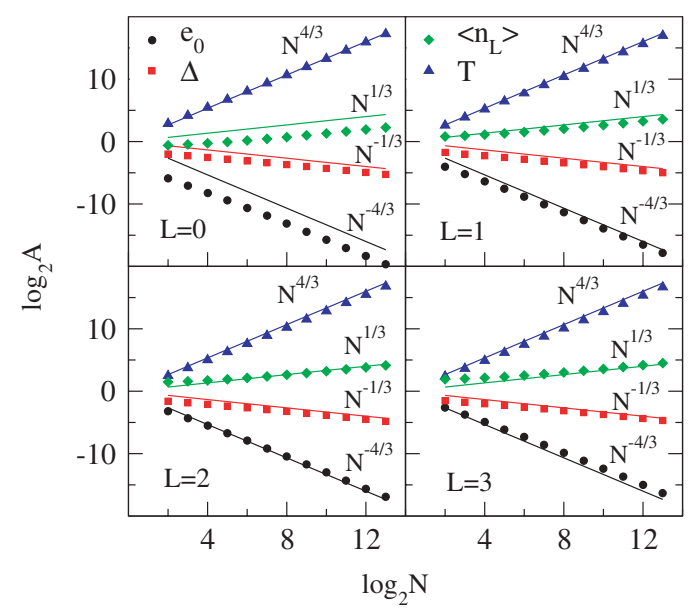

FIG. 16. (Color online) Plot of the singular parts of $e_{0}, \Delta,\left\langle n_{L}\right\rangle$, and $T$ at the critical point $x_{c}=1 / 2$ as a function of the boson number $N$ for different values of $L$. 
In this figure, only the singular part of the physical quantities of interest is plotted; the regular one is removed using the ad hoc expressions given in this work. As can be seen, the exponents are independent of $L$ as expected from our calculations and match very well the predicted values.

For $L=0$, these exponents can also be obtained by noting that the LMG model can be seen as an Ising model in a transverse field with long-range interactions $[42,43]$. Then, the scaling variable $N \Xi^{3 / 2}$ is obtained from the upper critical dimension of the equivalent model with short-range interactions that are known to be $d_{c}=3$ in this case. For $L \neq 0$, the two-level system studied in this article cannot be simply mapped onto a short-range interaction model. Thus, it is rather a remarkable fact that the finite-size scaling exponents are independent of $L$. However, as explained in Sec. VI, this is because the $\beta^{4}$-like potential underlying the critical theory.

\section{SUMMARY AND CONCLUSIONS}

In this article, we have studied two-level boson models where the lower boson has a zero angular momentum ( $s$ boson) and the upper one an angular momentum $L$. All these models are defined by the $U(2 L+2)$ algebra, from which one can find chains of subalgebras going down to the $O(3)$ angular momentum algebra. When the Hamiltonian is written as a combination of Casimir operators of a chain of subalgebras, it is said that a dynamical symmetry occurs and the problem is analytically solvable. In this article, we focused on the study of the quantum phase transition that appears when the boson system has a $O(2 L+1)$ symmetry, i.e., a transition from $U(2 L+1)$ to $O(2 L+2)$ dynamical symmetries. This second-order transition is well described by a mean-field approach and the subtleties arise in the finite-size corrections. Here, we have explicitly computed these corrections for several physical quantities using, first, a $1 / N$ expansion naturally given by the Holstein-Primakoff representation of the angular momenta, and, second, the continuous unitary transformations to diagonalize the Hamiltonian. In the spherical (symmetric) phase, we have thus been able to capture corrections beyond the standard random phase approximation and to show that the $1 / N$ expansion is singular at the critical point. The analysis of these singularities has allowed us to compute the finite-size scaling exponents that have been found to be independent of $L$. In the deformed (broken) phase, we have only computed the first corrections via a simple Bogoliubov transformations, to show the main difference with the spherical one.

We have also presented a formalism based on boson seniority that provides a simple and efficient way of solving numerically the problem for a large number of bosons (a few thousands). Using this powerful algorithm, we have compared order by order analytical and numerical results and found an excellent agreement between both. We hope that the present work will help in understanding the approach to the macroscopic limit in such models, a problem that has recently drawn much attention $[19,40]$.

\section{ACKNOWLEDGMENTS}

We thank J.-M. Maillard and D. Mouhanna for fruitful and stimulating discussions. S. Dusuel gratefully acknowledges financial support of the DFG in SP1073. This work has been partially supported by the Spanish DGI under projects FIS2005-01105, BFM2003-05316-C02-02, BFM2003-05316, and FPA2003-05958.

\section{APPENDIX A: FLOW EQUATIONS FOR THE HAMILTONIAN IN THE SYMMETRIC PHASE}

In this appendix, we give at each order the flow equations for the couplings and the initial conditions obtained from the $1 / N$ expansion of the Hamiltonian [Eq. (43)], For clarity, we have not explicitly written the $l$ dependence of all functions $h_{k, \alpha, \beta}^{(\delta)}$.

\section{Order $(1 / N)^{-1}$}

At this order, one has one flow equation

$$
\partial_{l} h_{0,0,0}^{(0)}=0
$$

with

$$
h_{0,0,0}^{(0)}(0)=\frac{1-x}{4}
$$

\section{Order $(1 / N)^{0}$}

At this order, one has three flow equations

$$
\begin{aligned}
& \partial_{l} h_{0,0,0}^{(1)}=-4(2 L+1) h_{2,0,0}^{(0)}{ }^{2}, \\
& \partial_{l} h_{0,1,0}^{(0)}=-8 h_{2,0,0}^{(0)}{ }^{2}, \\
& \partial_{l} h_{2,0,0}^{(0)}=-2 h_{0,1,0}^{(0)} h_{2,0,0}^{(0)},
\end{aligned}
$$

with

$$
\begin{aligned}
& h_{0,0,0}^{(1)}(0)=0, \\
& h_{0,1,0}^{(0)}(0)=\frac{3 x-1}{2}, \\
& h_{2,0,0}^{(0)}(0)=-\frac{1-x}{4} .
\end{aligned}
$$

\section{Order $(1 / N)^{1}$}

At this order, one has six flow equations

$$
\begin{aligned}
\partial_{l} h_{0,0,0}^{(2)}= & -8(2 L+1) h_{2,0,0}^{(0)} h_{2,0,0}^{(1)}, \\
\partial_{l} h_{0,1,0}^{(1)}=- & -8 h_{2,0,0}^{(0)}\left[2 h_{2,0,0}^{(1)}+(2 L+3) h_{2,1,0}^{(0)}\right], \\
\partial_{l} h_{2,0,0}^{(1)}=- & -2\left\{h_{0,1,0}^{(0)} h_{2,0,0}^{(1)}+h_{2,0,0}^{(0)}\right. \\
\times & {\left.\left[h_{0,1,0}^{(1)}+h_{0,2,0}^{(0)}+(2 L+1) h_{0,0,1}^{(0)}\right]\right\}, } \\
& \partial_{l} h_{0,2,0}^{(0)}=-16 h_{2,0,0}^{(0)} h_{2,1,0}^{(0)}, \\
& \partial_{l} h_{0,0,1}^{(0)}=-8 h_{2,0,0}^{(0)} h_{2,1,0}^{(0)},
\end{aligned}
$$


$\partial_{l} h_{2,1,0}^{(0)}=-2\left[h_{0,1,0}^{(0)} h_{2,1,0}^{(0)}+2 h_{2,0,0}^{(0)}\left(h_{0,2,0}^{(0)}+h_{0,0,1}^{(0)}\right)\right]$,

with

$$
\begin{aligned}
h_{0,0,0}^{(2)}(0) & =0 \\
h_{0,1,0}^{(1)}(0) & =0 \\
h_{2,0,0}^{(1)}(0) & =-\frac{1-x}{8}, \\
h_{0,2,0}^{(0)}(0) & =\frac{1-x}{4}, \\
h_{0,0,1}^{(0)}(0) & =\frac{1-x}{4}, \\
h_{2,1,0}^{(0)}(0) & =\frac{1-x}{4} .
\end{aligned}
$$

\section{Order $(1 / N)^{2}$}

At this order, one has 10 flow equations

$$
\begin{aligned}
\partial_{l} h_{0,0,0}^{(3)}= & -4(2 L+1)\left(h_{2,0,0}^{(1)}{ }^{2}+2 h_{2,0,0}^{(0)} h_{2,0,0}^{(2)}\right), \\
\partial_{l} h_{0,1,0}^{(2)}= & -8\left[h_{2,0,0}^{(1)}{ }^{2}+2 h_{2,0,0}^{(0)} h_{2,0,0}^{(2)}+(2 L+3)\right. \\
& \left.\times\left(h_{2,0,0}^{(0)} h_{2,1,0}^{(1)}+h_{2,0,0}^{(1)} h_{2,1,0}^{(0)}+\frac{1}{2} h_{2,1,0}^{(0)}{ }^{2}\right)\right], \\
\partial_{l} h_{2,0,0}^{(2)}= & -2\left\{h_{2,0,0}^{(0)}\left[h_{0,1,0}^{(2)}+h_{0,2,0}^{(1)}+(2 L+1) h_{0,0,1}^{(1)}\right]\right. \\
& +h_{2,0,0}^{(1)}\left[h_{0,1,0}^{(1)}+h_{0,2,0}^{(0)}+(2 L+1) h_{0,0,1}^{(0)}\right] \\
& \left.+h_{2,0,0}^{(2)} h_{0,1,0}^{(0)}\right\}, \\
\partial_{l} h_{0,2,0}^{(1)}= & -8\left[2\left(h_{2,0,0}^{(0)} h_{2,1,0}^{(1)}+h_{2,0,0}^{(1)} h_{2,1,0}^{(0)}\right)\right. \\
& \left.+(2 L+5) h_{2,0,0}^{(0)} h_{2,2,0}^{(0)}\right]-4(2 L+7) h_{2,1,0}^{(0)}{ }^{2},
\end{aligned}
$$

$$
\begin{aligned}
\partial_{l} h_{0,0,1}^{(1)}= & -8\left[h_{2,0,0}^{(0)} h_{2,1,0}^{(1)}+h_{2,0,0}^{(1)} h_{2,1,0}^{(0)}+h_{2,0,0}^{(0)} h_{2,2,0}^{(0)}+h_{2,1,0}^{(0)} 2\right. \\
& \left.+2(2 L+3) h_{2,0,0}^{(0)} h_{2,0,1}^{(0)}\right],
\end{aligned}
$$

$$
\begin{aligned}
\partial_{l} h_{2,1,0}^{(1)}= & -2\left\{h _ { 2 , 0 , 0 } ^ { ( 0 ) } \left[2\left(h_{0,2,0}^{(1)}+h_{0,0,1}^{(1)}\right)+3 h_{0,3,0}^{(0)}\right.\right. \\
& \left.+(2 L+3) h_{0,1,1}^{(0)}\right]+2 h_{2,0,0}^{(1)}\left(h_{0,2,0}^{(0)}+h_{0,0,1}^{(0)}\right) \\
& +h_{2,1,0}^{(0)}\left[h_{0,1,0}^{(1)}+3 h_{0,2,0}^{(0)}+(2 L+3) h_{0,0,1}^{(0)}\right] \\
& \left.+h_{0,1,0}^{(0)} h_{2,1,0}^{(1)}\right\},
\end{aligned}
$$

$\partial_{l} h_{0,3,0}^{(0)}=-8\left(h_{2,1,0}^{(0)}{ }^{2}+2 h_{2,0,0}^{(0)} h_{2,2,0}^{(0)}\right)$,

$\partial_{l} h_{0,1,1}^{(0)}=-8\left(h_{2,1,0}^{(0)}{ }^{2}+2 h_{2,0,0}^{(0)} h_{2,2,0}^{(0)}+4 h_{2,0,0}^{(0)} h_{2,0,1}^{(0)}\right)$,

$\partial_{l} h_{2,2,0}^{(0)}=-2\left[h_{2,0,0}^{(0)}\left(3 h_{0,3,0}^{(0)}+2 h_{0,1,1}^{(0)}\right)\right.$

$$
\left.+2 h_{2,1,0}^{(0)}\left(h_{0,2,0}^{(0)}+h_{0,0,1}^{(0)}\right)+h_{0,1,0}^{(0)} h_{2,2,0}^{(0)}\right]
$$

$\partial_{l} h_{2,0,1}^{(0)}=-2\left(h_{2,0,0}^{(0)} h_{0,1,1}^{(0)}+h_{0,1,0}^{(0)} h_{2,0,1}^{(0)}\right)$,

with

$$
\begin{aligned}
& h_{0,0,0}^{(3)}(0)=0, \\
& h_{0,1,0}^{(2)}(0)=0, \\
& h_{2,0,0}^{(2)}(0)=-\frac{3(1-x)}{32},
\end{aligned}
$$

$$
\begin{aligned}
& h_{0,2,0}^{(1)}(0)=\frac{1-x}{4}, \\
& h_{0,0,1}^{(1)}(0)=\frac{1-x}{4}, \\
& h_{2,1,0}^{(1)}(0)=\frac{1-x}{4} \\
& h_{0,3,0}^{(0)}(0)=0, \\
& h_{0,1,1}^{(0)}(0)=0, \\
& h_{2,2,0}^{(0)}(0)=0, \\
& h_{2,0,1}^{(0)}(0)=0 .
\end{aligned}
$$

\section{APPENDIX B: FLOW EQUATIONS FOR $b_{\mu}^{\dagger}$ IN THE SYMMETRIC PHASE}

In this appendix, we give at each order the flow equations for the couplings involved in the $1 / N$ expansion of $b_{\mu}^{\dagger}(l)$ [see Eq. (74)] and the corresponding initial conditions. For clarity, we have not explicitly written the $l$ dependence of all functions. Further, it is convenient to introduce $F_{s}=\frac{1}{2}\left(F_{+}+F_{-}\right)$and $F_{d}=\frac{1}{2}\left(F_{+}-F_{-}\right)$, for each function $F=A, B, C, D$.

\section{Order $(1 / N)^{0}$}

At this order, one has two flow equations

$$
\begin{aligned}
& \partial_{l} A_{s}^{(0)}=-2 h_{2,0,0}^{(0)} A_{s}^{(0)}, \\
& \partial_{l} A_{d}^{(0)}=2 h_{2,0,0}^{(0)} A_{d}^{(0)},
\end{aligned}
$$

with

$$
A_{s}^{(0)}(0)=A_{d}^{(0)}(0)=1 / 2
$$

\section{Order $(1 / N)^{1}$}

At this order, one has eight flow equations which decouples in two sets of four equations.

$$
\begin{aligned}
\partial_{l} A_{s}^{(1)}= & -2 h_{2,0,0}^{(0)}\left[A_{s}^{(1)}+B_{s}^{(0)}+(2 L+1) C_{s}^{(0)}+(2 L+3) D_{s}^{(0)}\right] \\
& -2 h_{2,0,0}^{(1)} A_{s}^{(0)},
\end{aligned}
$$

$\partial_{l} B_{s}^{(0)}=-2 h_{2,0,0}^{(0)}\left[B_{s}^{(0)}+2\left(C_{s}^{(0)}+D_{s}^{(0)}\right)\right]-2 h_{2,1,0}^{(0)} A_{s}^{(0)}$,

$\partial_{l} C_{s}^{(0)}=-2 h_{2,0,0}^{(0)}\left(B_{s}^{(0)}+D_{s}^{(0)}\right)-h_{2,1,0}^{(0)} A_{s}^{(0)}$,

$\partial_{l} D_{s}^{(0)}=-2 h_{2,0,0}^{(0)}\left(B_{s}^{(0)}+C_{s}^{(0)}\right)+h_{2,1,0}^{(0)} A_{s}^{(0)}$,

$\partial_{l} A_{d}^{(1)}=2 h_{2,0,0}^{(0)}\left[A_{d}^{(1)}+B_{d}^{(0)}+(2 L+1) C_{d}^{(0)}-(2 L+3) D_{d}^{(0)}\right]$ $+2 h_{2,0,0}^{(1)} A_{d}^{(0)}$,

$\partial_{l} B_{d}^{(0)}=2 h_{2,0,0}^{(0)}\left[B_{d}^{(0)}+2\left(C_{d}^{(0)}-D_{d}^{(0)}\right)\right]+2 h_{2,1,0}^{(0)} A_{d}^{(0)}$,

$\partial_{l} C_{d}^{(0)}=2 h_{2,0,0}^{(0)}\left(B_{d}^{(0)}-D_{d}^{(0)}\right)+h_{2,1,0}^{(0)} A_{d}^{(0)}$,

$\partial_{l} D_{s}^{(0)}=-2 h_{2,0,0}^{(0)}\left(B_{d}^{(0)}+C_{d}^{(0)}\right)+h_{2,1,0}^{(0)} A_{d}^{(0)}$,

with $\quad A_{s}^{(1)}(0)=B_{s}^{(0)}=C_{s}^{(0)}=D_{s}^{(0)}=A_{d}^{(1)}=B_{d}^{(0)}=C_{d}^{(0)}=$ $D_{d}^{(0)}(0)=0$. 


\section{APPENDIX C: SOLVING THE FLOW EQUATIONS}

The flow equations given in the above appendices have to be solved order by order in $1 / N$. At order $(1 / N)^{-1}$, nothing has to be done, so let us turn to order $(1 / N)^{0}$. The equations for $h_{0,1,0}^{(0)}(l)$ and $h_{2,0,0}^{(0)}(l)$ are easily solved by noticing that $h_{0,1,0}^{(0)}(l)^{2}-4 h_{2,0,0}^{(0)}(l)^{2}$ is a constant of the flow. One gets the hyperbolic solutions

$$
\begin{gathered}
h_{0,1,0}^{(0)}(l)=\frac{\Delta_{\infty}}{\tanh \left[2 \Delta_{\infty}\left(l+l_{0}\right)\right]}, \\
h_{2,0,0}^{(0)}(l)=\frac{-\operatorname{sgn}(\varepsilon) \Delta_{\infty}}{2 \sinh \left[2 \Delta_{\infty}\left(l+l_{0}\right)\right]} .
\end{gathered}
$$

We have denoted $\Delta_{\infty}$ the gap of the system at the thermodynamical limit, that is $h_{0,1,0}^{(0)}(\infty)$ [see Eq. (63)]. The quantity $l_{0}$ is such that the initial conditions are fulfilled, namely $h_{0,1,0}^{(0)}(0)=\Delta_{\infty} / \tanh \left[2 \Delta_{\infty} l_{0}\right]$, and we also introduced $\varepsilon=-h_{2,0,0}^{(0)}(0) /\left[2 h_{0,1,0}^{(0)}(0)\right]$. As already explained in Ref. [24], the best way to solve the flow equations is in fact to introduce a new "time scale" that is more adapted to the problem and defined by the following:

$$
t=\operatorname{sgn}(\varepsilon) \exp \left[2 \Delta_{\infty}\left(l+l_{0}\right)\right]
$$

with initial conditions now given at

$$
t_{0}=\operatorname{sgn}(\varepsilon) \exp \left(2 \Delta_{\infty} l_{0}\right) .
$$

After some algebra $t_{0}$ can also be shown to be equal to

$$
t_{0}=\frac{1}{\varepsilon}\left(1+\sqrt{1-\varepsilon^{2}}\right) .
$$

Eqs. (C1) and (C2) now read as follows:

$$
\begin{aligned}
& h_{0,1,0}^{(0)}(t)=\Delta_{\infty} \frac{t^{2}+1}{t^{2}-1}, \\
& h_{2,0,0}^{(0)}(t)=-\Delta_{\infty} \frac{t}{t^{2}-1},
\end{aligned}
$$

which are rational expressions in $t$. The renormalized values at $l \rightarrow \infty$ are now found by taking the limit $t \rightarrow t_{\infty}=\operatorname{sgn}(\varepsilon) \infty$. Let us remark that the off-diagonal coupling $h_{2,0,0}^{(0)}(t)$ goes to zero and behaves like $t^{-1}$ for $t \rightarrow t_{\infty}$. This will be true for all off-diagonal couplings creating two excitations because the energy cost of such excitations, in the thermodynamic limit and for large $t$, is nothing but $2 \Delta_{\infty}$, so that the couplings must vanish as $\exp \left(-2 \Delta_{\infty} l\right)$.

The last flow equation (for the spectrum) at order $(1 / N)^{0}$ is solved by noticing that $2 h_{0,0,0}^{(1)}(t)-(2 L+1) h_{0,1,0}^{(0)}(t)$ is a constant of the flow, equal to its initial value, namely $-(2 L+1)(3 x-1) / 2$.

The last task at order $(1 / N)^{0}$ is to obtain the solution for the observable. For this, one simply has to insert Eq. (C7) into Eqs. (B1) and (B2) and then replace $\partial_{l}$ with $2 \Delta_{\infty} t \partial_{t}$ and solve the resulting equation. This yields

$$
\begin{aligned}
& A_{s}^{(0)}(t)=\frac{1}{2} \sqrt{\frac{(t-1)\left(t_{0}+1\right)}{(t+1)\left(t_{0}-1\right)}}, \\
& A_{d}^{(0)}(t)=\frac{1}{2} \sqrt{\frac{(t+1)\left(t_{0}-1\right)}{(t-1)\left(t_{0}+1\right)}} .
\end{aligned}
$$

The next orders are solved in the same fashion: one inserts the expressions known from the previous orders, replaces $\partial_{l}$ with $2 \Delta_{\infty} t \partial_{t}$, and solves the equations (which is most simply achieved thanks to a computer algebra program). We refer the interested reader to the details given in Ref. [24], where some more technical details are given.

Let us emphasize that the usefulness of the $t$ variable comes from the fact that there exists only one basic energy scale in the problem, namely the gap. All energy scales are integer multiples of this gap. As previously mentioned, off-diagonal couplings associated to an energy scale $2 \Delta_{\infty}$ decay as $t^{-1}$, and in the general case, an off-diagonal coupling whose energy scale is $n \Delta_{\infty}$ decay as $t^{-n / 2}$. Such a time variable would be useless in a problem where many different energy scales exist.
[1] E. T. Jaynes and F. W. Cummings, Proc. IEEE 51, 89 (1963).

[2] F. Iachello and R. D. Levine, Algebraic Theory of Molecules (Oxford University Press, Oxford, 1995).

[3] H. Suhl, B. T. Matthias, and L. Walker, Phys. Rev. Lett. 3, 552 (1959).

[4] J. Högaasen-Feldman, Nucl. Phys. 28, 258 (1961).

[5] H. J. Lipkin, N. Meshkov, and A. J. Glick, Nucl. Phys. 62, 188 (1965).

[6] N. Meshkov, A. J. Glick, and H. J. Lipkin, Nucl. Phys. 62, 199 (1965).

[7] A. J. Glick, H. J. Lipkin, and N. Meshkov, Nucl. Phys. 62, 211 (1965).

[8] F. Iachello and A. Arima, The Interacting Boson Model (Cambridge University Press, Cambridge, 1987).

[9] J. Vidal, G. Palacios, and R. Mosseri, Phys. Rev. A 69, 022107 (2004).

[10] J. Vidal, R. Mosseri, and J. Dukelsky, Phys. Rev. A 69, 054101 (2004).
[11] J. Vidal, G. Palacios, and C. Aslangul, Phys. Rev. A 70, 062304 (2004).

[12] J. I. Latorre, R. Orús, E. Rico, and J. Vidal, Phys. Rev. A 71, 064101 (2005).

[13] F. Leyvraz and W. D. Heiss, Phys. Rev. Lett. 95, 050402 (2005).

[14] S. Dusuel and J. Vidal, Phys. Rev. Lett. 93, 237204 (2004).

[15] R. G. Unanyan, C. Ionescu, and M. Fleischhauer, Phys. Rev. A 72, 022326 (2005)

[16] H.-Q. Zhou, J. Links, R. H. McKenzie, and X.-W. Guan, J. Phys. A 36, L113 (2003).

[17] F. Pan, X. Zhang, and J. P. Draayer, J. Phys. A 35, 7173 (2002).

[18] R. W. Richardson, J. Math. Phys. 9, 1327 (1968).

[19] F. Iachello and N. V. Zamfir, Phys. Rev. Lett. 92, 212501 (2004).

[20] F. Pan and J. P. Draayer, Nucl. Phys. A636, 156 (1998).

[21] D. J. Rowe, Nucl. Phys. A745, 47 (2004).

[22] J. E. García-Ramos, J. Dukelsky, and J. M. Arias, Phys. Rev. C 72, 037301 (2005). 
[23] J. M. Arias, C. E. Alonso, A. Vitturi, J. E. García-Ramos, J. Dukelsky, and A. Frank, Phys. Rev. C 68, 041302(R) (2003). [24] S. Dusuel and J. Vidal, Phys. Rev. B 71, 224420 (2005).

[25] T. Holstein and H. Primakoff, Phys. Rev. 58, 1098 (1940).

[26] A. Klein and E. R. Marshalek, Rev. Mod. Phys. 63, 375 (1991).

[27] J. Dukelsky, G. G. Dussel, R. P. J. Perazzo, S. L. Reich, and H. M. Sofia, Nucl. Phys. A425, 93 (1984).

[28] D. J. Rowe, Phys. Rev. Lett. 93, 122502 (2004).

[29] F. Wegner, Ann. Phys. (Leipzig) 3, 77 (1994).

[30] S. D. Głazek and K. G. Wilson, Phys. Rev. D 48, 5863 (1993).

[31] S. D. Głazek and K. G. Wilson, Phys. Rev. D 49, 4214 (1994).

[32] F. Wegner, Phys. Rep. 348, 77 (2001).

[33] B. H. Bartlett, Master Thesis (2003), nucl-th/0305052.
[34] A. Mielke, Eur. Phys. J. B 5, 605 (1998).

[35] C. Knetter and G. S. Uhrig, Eur. Phys. J. B 13, 209 (2000).

[36] J. Stein, J. Phys. G 26, 377 (2000).

[37] S. Dusuel, J. Vidal, J. M. Arias, J. Dukelsky, and J. E. GarcíaRamos, Phys. Rev. C 72, 011301(R) (2005).

[38] R. W. Richardson, J. Math. Phys. 18, 1802 (1977).

[39] S. Dusuel and J. Vidal, Phys. Rev. A 71, 060304(R) (2005).

[40] D. J. Rowe, P. S. Turner, and G. Rosensteel, Phys. Rev. Lett. 93, 232502 (2004).

[41] F. Pan, Y. Zhang, and J. P. Draayer, J. Phys. G 31, 1039 (2005).

[42] R. Botet, R. Jullien, and P. Pfeuty, Phys. Rev. Lett. 49, 478 (1982).

[43] R. Botet and R. Jullien, Phys. Rev. B 28, 3955 (1983). 